\title{
Uniform estimates for characteristics-mixed finite method for transient advection-dominated diffusion problems in two-dimensional space ${ }^{\frac{1}{2}}$
}

\author{
Chen Huanzhen ${ }^{\mathrm{a}, *}$, Gao Lei ${ }^{\mathrm{a}}$, Wang Hong ${ }^{\mathrm{b}}$ \\ ${ }^{a}$ School of Mathematical Sciences, Shandong Normal University, Jinan, \\ Shandong Province, 250014, China. \\ ${ }^{b}$ Department of Mathematics, University of South Carolina, Columbia, \\ South Carolina 29208, USA.
}

\begin{abstract}
We prove uniform error estimates for the characteristics-mixed finite element method for two-dimensional transient convection-dominated diffusion equations, in the sense that the generic constants in the error estimates depend linearly on certain Sobolev norms of the true solution but not on the scaling diffusion parameter $\varepsilon$. Numerical experiments are presented to confirm our theoretical findings.
\end{abstract}

Keywords: transient advection-dominated diffusion equations, characteristics-mixed finite element method, uniform error estimates, numerical experiments.

\section{Introduction}

We consider the following two-dimensional transient advection-diffusion equation

$$
\begin{array}{ll}
\text { (a) } \quad(\phi c)_{t}+\nabla \cdot(c \mathbf{u}-\varepsilon D \nabla c)=f_{1}-f_{2} c & \text { in } \Omega \times(0, T], \\
\text { (b) } c(\mathbf{x}, 0)=c_{0}(\mathbf{x}) & \text { in } \Omega .
\end{array}
$$

\footnotetext{
This work is supported in part by the National Natural Science Foundation of China under grant No. 10971254, 11301311, 11471196 and by the National Science Foundation under grant No. EAR-0934747 and DMS-1216923.

*Corresponding Author.

Email addresses: chhzh@sdnu.edu.cn (Chen Huanzhen), sunnydoll@sjtu.edu.cn (Gao Lei), hwang@math.sc.edu (Wang Hong)
}

Preprint submitted to Applied Mathematics and Computation December 24, 2015

(C) 2016. This manuscript version is made available under the Elsevier user license http://www.elsevier.com/open-access/userlicense/1.0/ 
The problem (1) describes phenomena such as the flow of heat within a moving fluid, the transport of dissolved nutrients or contaminants within groundwater flow, and the miscible displacement process of a surfactant in an incompressible hydrocarbon phase in a petroleum reservoir [see 8, 9]. Here $\Omega=[a, b] \times[c, d]$ is a rectangular domain, $\phi(\mathbf{x})$ is the porosity of the medium, $\mathbf{u}(\mathbf{x}, t)$ is the velocity of the flow, $D(\mathbf{x}, t)$ represents the diffusion tensor with $0<D_{\min } \leq D(\mathbf{x}, t) \leq D_{\max }<\infty$ for any $(\mathbf{x}, t) \in \Omega \times[0, T]$, $f_{1}(\mathbf{x}, t)$ and $f_{2}(\mathbf{x}, t) \geq 0$ account for external sources and sinks respectively. The parameter $\varepsilon \in(0,1)$ scales the diffusion and characterizes the advectiondominance of Eq. (1), $c$ is the $\varepsilon$-dependent concentration of a solute or solvent, $c_{0}$ is a prescribed initial data. We assume that problem (1) is closed by a no-flow boundary condition.

The diffusive coefficient $\varepsilon D$ is uniformly positive definite then problem (1) is strictly parabolic. However, in many applications $\varepsilon$ is small enough, thus advection dominates diffusion, the equation is nearly hyperbolic in nature and admits solutions with complex structures such as boundary and dynamic interior layers, and thus present serious mathematical and numerical difficulties [see 14]. So the traditional parabolic discretization schemes applied to the problem tend to introduce into the solution an excessive amount of numerical diffusion and spurious oscillation near the layers. The corresponding error estimates depend inversely on $\varepsilon$ and could blow up as $\varepsilon$ tends to zero.

The modified method of characteristics (MMOC) combined with the lowest-order mixed finite element method (the characteristics-mixed finite element method) proposed in Arbogast \& Wheeler [1] is a representative of Eulerian-Lagrangian methods, which provide an effective approach for numerically solving transient advection-diffusion equations. The method combines the advection and capacity terms in the governing equation to carry out the temporal discretization in a Lagrangian coordinate, and uses the lowestorder mixed finite element to carry out spatial discretization. It generates a accurate, element-by-element mass balanced numerical solution even if large time steps and coarse spatial meshes are used. However, the error estimates derived for the method depend still inversely on $\varepsilon$, which do not fully reflect the strength of the method observed numerically.

In this article, motivated by the idea for the Eulerian-Lagrangian localized adjoint method and the modified method of characteristics [see 16, 17, 19, 18], we prove error estimates for the characteristics-mixed finite element and its postprocessing schemes for Eq. (1) with a periodic boundary condition. In the error derivation the mixed elliptic projection is replaced by the interpo- 
lation operators, therefore $\varepsilon$-uniform optimal-order error estimates for the unknown function and its flux, and $\frac{3}{2}$-order error estimate for the postprocessing solution are derived. The generic constants in these estimates do not explicitly depend on $\varepsilon$, but depend linearly on certain Sobolev norms of the true solution.

The article is organized as follows. In Section 2, we revisit the characteristicsmixed finite element procedure and the locally postprocessing scheme. In Section 3, we present preliminary lemmas which are to be used in the convergence analysis. In Section 4 , we prove $\varepsilon$-uniform optimal-order error estimates for the mixed finite element solution and the postprocessing solution. In Section 5, we conduct numerical experiments to observe the convergence behavior of the numerical scheme.

\section{Revisit The Characteristics-mixed Finite Element Method}

Let $H^{s}(E), s \geq 0$, denote the usual Sobolev space provided the norm $\|\cdot\|_{s, E}$, and let $\|\cdot\|_{E}$ denote the corresponding $L^{2}$-norm. If $E=\Omega$ we omit this subscript. For any Banach space $X$, we use the following spaces involving time [see 7]

$$
\begin{aligned}
& W_{p}^{k}(0, T ; X)=\left\{v:\left\|\frac{\partial^{l} v}{\partial t^{l}}(\cdot, t)\right\|_{X} \in L^{p}(0, T), 0 \leq l \leq k, 1 \leq p \leq \infty\right\} \\
& \|v\|_{W_{p}^{k}(X)}= \begin{cases}\left(\sum_{l=1}^{k} \int_{0}^{T}\left\|\frac{\partial^{l} v}{\partial t^{l}}(\cdot, t)\right\|_{X}^{p} d t\right)^{\frac{1}{p}}, & 1 \leq p<\infty, \\
\max _{0 \leq l \leq k} \operatorname{ess} \sup _{t \in(0, T)}\left\|\frac{\partial^{l} v}{\partial t^{l}}(\cdot, t)\right\|_{X}, & p=\infty .\end{cases}
\end{aligned}
$$

If a function $f(x, t)$ is defined only at discrete time steps $t^{n}$, we understand that the function $f$ has been extended by constant to the time interval $\left(t^{n-1}, t^{n}\right]$. We also introduce Sobolev space that incorporate the no-flow boundary condition

$$
\mathbf{H}=\left\{\boldsymbol{\chi}: \boldsymbol{\chi} \in\left(L^{2}(\Omega)\right)^{2}, \nabla \cdot \chi \in L^{2}(\Omega), \boldsymbol{\chi} \cdot \mathbf{n}=0\right\}
$$

equipped with the norm and $\varepsilon$-weighted $L^{2}$-norm

$$
\|\chi\|_{\mathbf{H}}=\left(\|\chi\|^{2}+\|\operatorname{div} \chi\|^{2}\right)^{1 / 2}, \quad\|\chi\|_{L_{\varepsilon}^{2}(\Omega)}=\varepsilon^{-\frac{1}{2}}\|\chi\|,
$$


and

$$
\mathcal{W}=\left\{w \in L^{2}(\Omega): w \text { is piecewise constant }\right\} .
$$

In the derivation of the weak formulation of Eq.(1) we follow the line as in Arbogast \& Wheeler [1] by considering the advection diffusion equation in a space-time framework. Choose some domain $R \subset \Omega$ and two times $0 \leq t^{n-1}<t^{n} \leq T$. The hyperbolic part of the equation, $\phi c_{t}+u \cdot \nabla c$, defines the characteristics $\check{\mathbf{x}}(\mathbf{x}, t)$ along the interstitial velocity $\mathbf{v}=\mathbf{u} / \phi$ by

$$
\check{\mathbf{x}}_{t}=\mathbf{v}(\check{\mathbf{x}}, t), \quad t^{n-1} \leq t \leq t^{n} ; \quad \check{\mathbf{x}}\left(\mathbf{x}, t^{n}\right)=\mathbf{x} .
$$

Let $\check{R}(t)$ denote the trace-back of $R$ to time $t, \mathcal{R}=\{(\mathbf{x}, t) \in \Omega \times[0, T]$ : $\left.t^{n-1} \leq t \leq t^{n}, \mathbf{x} \in \check{R}(t)\right\}$ denote the space-time region that follows the characteristics.

We define the approximate characteristics

$$
\check{\mathbf{x}}_{n}(\mathbf{x}, t)=\mathbf{x}-\mathbf{v}\left(\mathbf{x}, t^{n}\right)\left(t^{n}-t\right),
$$

which is a one-to-one mapping from $\bar{\Omega}$ into $\bar{\Omega}$, whose inverse is called $\hat{\mathbf{x}}_{n}(\cdot, t)$. For a function $\phi(\mathbf{x}, t), t^{n-1}<t \leq t^{n}$, we let

$$
\check{\phi}(\mathbf{x}, t)=\phi\left(\check{\mathbf{x}}_{n}(\mathbf{x}, t), t\right) \quad \text { and } \quad \hat{\phi}(\mathbf{x}, t)=\phi\left(\hat{\mathbf{x}}_{n}(\mathbf{x}, t), t^{n}\right) .
$$

Then $\hat{\phi}\left(\mathbf{x}, t^{n-1,+}\right)=\hat{\phi}^{n-1,+}(\mathbf{x})$ follows the approximate characteristics forward from time $t^{n-1}$ to $t^{n}$ to become $\phi^{n}(\mathbf{x})$.

Introducing the diffusive flux $\mathbf{z}=-\varepsilon D \nabla c$, Eq.(1) is rewritten as a first order system,

$$
\begin{array}{ll}
\text { (a) } \quad(\phi c)_{t}+\nabla \cdot(c \mathbf{u}+\mathbf{z})=f_{1}-f_{2} c & \text { in } \Omega \times[0, T], \\
\text { (b) } \quad \mathbf{z}=-\varepsilon D \nabla c & \text { in } \Omega \times[0, T], \\
\text { (c) } \quad c(\mathbf{x}, 0)=c_{0}(\mathbf{x}), & \mathbf{x} \in \Omega .
\end{array}
$$

Multiplying (4a) by $\hat{w}(\mathbf{x}, t), w \in \mathcal{W}$, integrating by parts over the space-time region $\mathcal{R}$ and summing the resulting integrals over $\Omega$; Multiplying (4b) by $\chi \in \mathbf{H}$ and integrating by parts over $\Omega$, then noticing the assumption of the no-flow and periodic boundary condition, we derive the mixed variational 
formulation corresponding to (1) as to find $(\mathbf{z}, c) \in \mathbf{H} \times \mathcal{W}$ such that

(a) $\left(\phi^{n} c^{n}, w\right)-\left(\phi^{n-1} c^{n-1}, \hat{w}^{n-1,+}\right)+\int_{t^{n-1}}^{t^{n}}(\nabla \cdot \mathbf{z}, \hat{w}) d t$ $=\int_{t^{n-1}}^{t^{n}}\left\{\left(f_{1}-f_{2} c, \hat{w}\right)+(\nabla \cdot[(\hat{\mathbf{u}}-\mathbf{u}) c], \hat{w})\right\} d t, \quad w \in \mathcal{W}$

(b) $\left(D^{-1} \mathbf{z}, \boldsymbol{\chi}\right)=\varepsilon(c, \nabla \cdot \boldsymbol{\chi}), \quad \boldsymbol{\chi} \in \mathbf{H}$,

(c) $c(\mathbf{x}, 0)=c_{0}(\mathbf{x}), \quad \mathbf{x} \in \Omega$.

We define a uniform space-time partition on $\Omega \times[0, T]$ by $x_{i}=a+i \Delta x$ for $i=0,1, \ldots, I$ with $\Delta x:=(b-a) / I ; y_{j}=c+j \Delta y$ for $j=0,1, \ldots, J$ with $\Delta y:=(d-c) / J$, and $t^{n}=n \Delta t$ for $n=0,1, \ldots, N$ with $\Delta t:=T / N$. Let $\Omega_{i, j}=\left(x_{i-1}, x_{i}\right) \times\left(y_{j-1}, y_{j}\right), h=\sqrt{(\Delta x)^{2}+(\Delta y)^{2}}, x_{i-\frac{1}{2}}=\left(x_{i-1}+x_{i}\right) / 2$ and $y_{j-\frac{1}{2}}=\left(y_{j-1}+y_{j}\right) / 2$. In this paper, we use the lowest-order RaviartThomas mixed rectangular finite element spaces [see 13] $\mathbf{H}_{h} \times V_{h} \subset \mathbf{H} \times \mathcal{W}$ to approximate the problem (1). For postprocessing we need to define a space $\tilde{V}_{h}$ consisting of functions that are discontinuous and piecewise bilinear over the space partition.

We define the characteristic-mixed finite element scheme from (5) as to find $\left(\mathbf{z}_{h}^{n}, c_{h}^{n}\right) \in \mathbf{H}_{h} \times V_{h}$, satisfying

$$
\begin{aligned}
& (a)\left(\phi^{n} c_{h}^{n}, w_{h}\right)-\left(\phi^{n-1} \tilde{c}_{h}^{n-1}, \hat{w}_{h}^{n-1,+}\right)+\varepsilon\left(\nabla \cdot \mathbf{z}_{h}^{n}, w_{h}\right) \Delta t^{n} \\
& =\int_{t^{n-1}}^{t^{n}}\left(f_{1}-f_{2} \hat{c}_{h}, \hat{w}_{h}\right) d t, \quad w \in V_{h}, \\
& \text { (b) }\left(D_{n}^{-1} \mathbf{z}_{h}^{n}, \boldsymbol{\chi}_{h}\right)=\varepsilon\left(c_{h}^{n}, \nabla \cdot \boldsymbol{\chi}_{h}\right), \quad \chi \in \mathbf{H}_{h}, \\
& \text { (c) } c_{h}(\mathbf{x}, 0)=c_{0 h}(\mathbf{x}), \quad \mathbf{x} \in \Omega \text {. }
\end{aligned}
$$

Where $D_{n}^{-1}=: D^{-1}\left(\mathbf{x}, t^{n}\right) \geq \alpha_{0}>0$.

We then locally postprocess the concentration by finding $\tilde{c}_{h}^{n} \in \tilde{V}_{h}$ such that on each element $\Omega_{i, j}$,

$$
\begin{aligned}
& (a)\left(\phi^{n}\left(\tilde{c}_{h}^{n}-c_{h}^{n}\right), 1\right)_{\Omega_{i, j}}=0, \\
& (b)\left(\varepsilon D^{n} \nabla \tilde{c}_{h}^{n}+\mathbf{z}_{h}^{n}, \nabla w\right)_{\Omega_{i, j}}=0, \quad w \in \tilde{V}_{h} .
\end{aligned}
$$

The solvability of the discrete problem (6) and the postprocessing scheme (7) is given by Lemma 1 in Arbogast \& Wheeler [1].

We end this section by some remarks on the boundary conditions. The noflow and the periodic boundary conditions are used in (5) only for simplifying 
the numerical analysis. For implementation the no-flow boundary condition can be replaced by one of three boundary conditions: Dirichlet, in-flow and out-flow, and the periodic boundary condition may not be prescribed, see (2.7)-(2.9) in Arbogast \& Wheeler [1].

\section{Preliminaries}

Since the elliptic projection used in traditional error analysis is the main fact that produces $\varepsilon$-dependent error estimates we shall define the interpolations to replace of the mixed elliptic projection and present their wellestablished properties in this section.

\subsection{Interpolation for Concentration}

We assume $\mathcal{Q}_{r}\left(\Omega_{i, j}\right)$ denotes the space of polynomials of degree less than or equal to $r(r=0,1)$ in each space direction. Then define the constant interpolation $R_{h}: C\left(\bar{\Omega}_{i, j}\right) \rightarrow \mathcal{Q}_{0}\left(\Omega_{i, j}\right)$,

$$
R_{h} v(x, y)=v\left(x_{i-\frac{1}{2}}, y_{j-\frac{1}{2}}\right), \quad 1 \leq i \leq I, \quad 1 \leq j \leq J
$$

and the bilinear interpolation $\tilde{R}_{h}: C\left(\bar{\Omega}_{i, j}\right) \rightarrow \mathcal{Q}_{1}\left(\Omega_{i, j}\right)$

$$
\tilde{R}_{h} v\left(x_{i}, y_{j}\right)=v\left(x_{i}, y_{j}\right), \quad 1 \leq i \leq I, \quad 1 \leq j \leq J .
$$

The following estimates are well known [see 4, 5]

$$
\begin{aligned}
& \left\|R_{h} v-v\right\|_{k, \Omega_{i, j}} \leq Q h^{1-k}\|v\|_{1, \Omega_{i, j}}, \quad \forall v \in H^{1}\left(\Omega_{i, j}\right), \quad k=0,1, \\
& \left\|\tilde{R}_{h} v-v\right\|_{k, \Omega_{i, j}} \leq Q h^{2-k}\|v\|_{2, \Omega_{i, j}}, \quad \forall v \in H^{2}\left(\Omega_{i, j}\right), \quad k=0,1,
\end{aligned}
$$

Lemma 3.1. Let $u \in H^{2}(\Omega)$. Then the following integral expression holds

$$
\left(u-R_{h} u, w\right)_{\Omega_{i, j}} \leq Q h^{2}\|u\|_{2, \Omega_{i, j}}\|w\|_{\Omega_{i, j}}, \quad \forall w \in V_{h} .
$$

Proof By Taylor's expansions

$$
\begin{aligned}
\left.\left(R_{h}-I\right) v(x, y)\right|_{\bar{\Omega}_{i, j}}=\int_{x_{i-\frac{1}{2}}}^{x} v_{\alpha}(\alpha, y) d \alpha & \\
+ & \int_{y_{j-\frac{1}{2}}}^{y} v_{\beta}(x, \beta) d \beta+\int_{y_{j-\frac{1}{2}}}^{y} \int_{x_{i-\frac{1}{2}}}^{x} v_{\alpha \beta}(\alpha, \beta) d \alpha d \beta
\end{aligned}
$$


we have

$$
\begin{aligned}
(u- & \left.R_{h} u, w\right)_{\Omega_{i, j}}=-\int_{\Omega_{i, j}} w \int_{x_{i-\frac{1}{2}}}^{x} u_{\alpha}(\alpha, y) d \alpha d x d y \\
& -\int_{\Omega_{i, j}} w \int_{y_{j-\frac{1}{2}}}^{y} u_{\beta}(x, \beta) d \beta d x d y \\
& -\int_{\Omega_{i, j}} w \int_{y_{j-\frac{1}{2}}}^{y} \int_{x_{i-\frac{1}{2}}}^{x} u_{\alpha \beta}(\alpha, \beta) d \alpha d \beta d x d y .
\end{aligned}
$$

The third term on the right-hand side of (14) is bounded by using Hölder's inequality,

$$
\begin{aligned}
& \left|-\int_{\Omega_{i, j}} w \int_{y_{j-\frac{1}{2}}}^{y} \int_{x_{i-\frac{1}{2}}}^{x} u_{\alpha \beta}(\alpha, \beta) d \alpha d \beta d x d y\right| \\
& \quad \leq \int_{\Omega_{i, j}}|w|\left|\int_{y_{j-1}}^{y_{j}} \int_{x_{i-1}}^{x_{i}} u_{\alpha \beta}(\alpha, \beta) d \alpha d \beta\right| d x d y \\
& \quad \leq Q h^{2}\|w\|_{\Omega_{i, j}}\|u\|_{2, \Omega_{i, j}} .
\end{aligned}
$$

Noting that $w$ is a piecewise constant and then $\int_{x_{i-1}}^{x_{i}}\left(x-x_{i-\frac{1}{2}}\right) d x=0$, we have the following estimate for the first term on the right side of (14),

$$
\begin{aligned}
& \left|-\int_{\Omega_{i, j}} w \int_{x_{i-\frac{1}{2}}}^{x} u_{\alpha}(\alpha, y) d \alpha d x d y\right| \\
& =\left|\int_{\Omega_{i, j}} w \int_{x_{i-\frac{1}{2}}}^{x} \int_{x_{i-\frac{1}{2}}}^{\alpha} u_{\beta \beta}(\beta, y) d \beta d \alpha d x d y\right| \\
& \leq\left(\int_{\Omega_{i, j}}|w|^{2} d x d y\right)^{\frac{1}{2}}\left(\int_{\Omega_{i, j}}\left|\int_{y_{j-1}}^{y_{j}} \int_{x_{i-1}}^{x_{i}} u_{\alpha \beta}(\alpha, \beta) d \alpha d \beta\right|^{2} d x d y\right)^{\frac{1}{2}} \\
& \leq Q h^{2}\|u\|_{2, \Omega_{i, j}}\|w\|_{\Omega_{i, j}} .
\end{aligned}
$$

By symmetry we have the same estimate as (16) for the second term. Combining (15) and (16) finishes the proof of the lemma.

\subsection{Interpolation for Flux}

For a vector function $\mathbf{w}=\left(w_{1}, w_{2}\right)^{T} \in\left(C^{0}\left(\Omega_{i, j}\right)\right)^{2}$, we let

$$
\begin{array}{ll}
\bar{w}_{1}(x, y)=\bar{w}_{1}\left(x, y_{j}\right)=\frac{1}{\Delta y} \int_{y_{j-1}}^{y_{j}} w_{1}(x, y) d y, & x_{i-1} \leq x \leq x_{i}, \\
\bar{w}_{2}(x, y)=\bar{w}_{2}\left(x_{i}, y\right)=\frac{1}{\Delta x} \int_{x_{i-1}}^{x_{i}} w_{2}(x, y) d x, & y_{j-1} \leq y \leq y_{j},
\end{array}
$$


and define a local interpolation operator $\Pi_{i j} \mathbf{w}=\left(\Pi_{i x} w_{1}, \Pi_{j y} w_{2}\right)^{T}$ by [see 15]

$$
\begin{array}{ll}
\Pi_{i x} w_{1}(x, y)=\frac{x_{i}-x}{\Delta x} \bar{w}_{1}\left(x_{i-1}, y_{j}\right)+\frac{x-x_{i-1}}{\Delta x} \bar{w}_{1}\left(x_{i}, y_{j}\right), \quad x_{i-1} \leq x \leq x_{i}, \\
\prod_{j y} w_{2}(x, y)=\frac{y_{j}-y}{\Delta y} \bar{w}_{2}\left(x_{i}, y_{j-1}\right)+\frac{y-y_{j-1}}{\Delta y} \bar{w}_{2}\left(x_{i}, y_{j}\right), \quad y_{j-1} \leq y \leq y_{j} .
\end{array}
$$

Then we define the global interpolation operator $\Pi_{h}:\left(C^{0}(\Omega)\right)^{2} \rightarrow \mathbf{H}_{h}$ by

$$
\left.\left(\Pi_{h} \mathbf{w}\right)\right|_{\Omega_{i, j}}=\Pi_{i, j} \mathbf{w} \text { for } 1 \leq i \leq I \text { and } 1 \leq j \leq J
$$

It is easily seen that the interpolation operator $\Pi_{h}$, which is piecewise constant in one direction and piecewise linear in the other direction, is welldefined. Other than restate its well-established approximation properties given in Rui [15]

$$
\left\|\Pi_{h} \mathbf{w}-\mathbf{w}\right\| \leq Q h\|\mathbf{w}\|_{1}, \quad\left(\nabla \cdot\left(\mathbf{w}-\Pi_{h} \mathbf{w}\right), p_{i j}\right)=0, \quad \forall p_{i j} \in \mathcal{Q}_{0}\left(\Omega_{i, j}\right),
$$

we shall further discover a better estimate in the following lemma.

Lemma 3.2. Let $\mathbf{w}=\left(w_{1}, w_{2}\right)^{T} \in\left(H^{2}(\Omega)\right)^{2}$. Then we have

$$
\left(D^{-1}\left(\mathbf{w}-\Pi_{h} \mathbf{w}\right), \boldsymbol{\chi}\right) \leq Q h^{2}\|\mathbf{w}\|_{2}\|\boldsymbol{\chi}\|, \quad \forall \boldsymbol{\chi} \in \mathbf{H}_{h}
$$

Its proof will be given in Appendix.

\subsection{Projection for Postprocessing}

We define $\tilde{C}_{h} \in \tilde{V}_{h}$ on each element $\Omega_{i, j} \in \mathcal{T}_{h}$

$$
\begin{aligned}
& \text { (a) }\left(\phi\left(\tilde{C}_{h}-R_{h} c\right), 1\right)_{\Omega_{i, j}}=0, \\
& \text { (b) }\left(\varepsilon D \nabla \tilde{C}_{h}+\Pi_{h} \mathbf{z}, \nabla w\right)_{\Omega_{i, j}}=0, \quad \forall w \in \tilde{V}_{h} .
\end{aligned}
$$

For simplicity, we assume that

$$
\begin{aligned}
& c-c_{h}=c-R_{h} c+R_{h} c-c_{h}=\rho+e, \\
& c-\tilde{c}_{h}=c-\tilde{C}_{h}+\tilde{C}_{h}-\tilde{c}_{h}=\tilde{\rho}+\tilde{e}, \\
& \mathbf{z}-\mathbf{z}_{h}=\mathbf{z}-\Pi_{h} \mathbf{z}+\Pi_{h} \mathbf{z}-\mathbf{z}_{h}=\boldsymbol{\eta}+\boldsymbol{\xi} .
\end{aligned}
$$

The following lemma gives the approximation properties of $\tilde{C}_{h}$. 
Lemma 3.3. Let $c$ be the solution of (5) and $\phi \in W_{\infty}^{1}(\Omega \times J)$. Then the following estimates hold

$$
\begin{aligned}
& \text { (a) }\left\|\nabla\left(c-\tilde{C}_{h}\right)\right\|_{\Omega_{i, j}} \leq Q h\|c\|_{2, \Omega_{i, j}}, \\
& \text { (b) }\left\|c-\tilde{C}_{h}\right\|_{\Omega_{i, j}} \leq Q h^{2}\|c\|_{2, \Omega_{i, j}}, \\
& \text { (c) }\left\|\left(c-\tilde{C}_{h}\right)_{t}\right\|_{\Omega_{i, j}} \leq Q h^{2}\left(\|c\|_{2, \Omega_{i, j}}+\left\|c_{t}\right\|_{2, \Omega_{i, j}}\right) .
\end{aligned}
$$

Proof Noting the definition of $\tilde{C}_{h}$ and $\mathbf{z}=-\varepsilon D \nabla c$ to form the error equation

$$
\begin{aligned}
& \text { (a) }(\phi \tilde{\rho}, 1)_{\Omega_{i, j}}=(\phi \rho, 1)_{\Omega_{i, j}}, \\
& \text { (b) } \varepsilon(D \nabla \tilde{\rho}, \nabla w)_{\Omega_{i, j}}=-(\boldsymbol{\eta}, \nabla w)_{\Omega_{i, j}}, \quad \forall w \in \tilde{V}_{h},
\end{aligned}
$$

in which (24b) is rewritten as

$$
\varepsilon(D \nabla \tilde{\rho}, \nabla \tilde{\rho})_{\Omega_{i, j}}=\varepsilon(D \nabla \tilde{\rho}, \nabla(\tilde{\rho}-w))_{\Omega_{i, j}}-(\boldsymbol{\eta}, \nabla w)_{\Omega_{i, j}} .
$$

Choosing $w=\tilde{R}_{h} c-\tilde{C}_{h}$ in (25) and combining (11) to have

$$
\begin{aligned}
\varepsilon(D \nabla \tilde{\rho}, & \nabla \tilde{\rho})_{\Omega_{i, j}}=\varepsilon\left(D \nabla \tilde{\rho}, \nabla\left(c-\tilde{R}_{h} c\right)\right)_{\Omega_{i, j}}-\left(\boldsymbol{\eta}, \nabla\left(\tilde{R}_{h} c-\tilde{C}_{h}\right)\right)_{\Omega_{i, j}} \\
& \leq \varepsilon Q h\|\nabla \tilde{\rho}\|_{\Omega_{i, j}}\|c\|_{2, \Omega_{i, j}}+Q h\|\mathbf{z}\|_{1, \Omega_{i, j}}\left\|\nabla\left(\tilde{R}_{h} c-\tilde{C}_{h}\right)\right\|_{\Omega_{i, j}} \\
& \leq \varepsilon Q h\|\nabla \tilde{\rho}\|_{\Omega_{i, j}}\|c\|_{2, \Omega_{i, j}}+\varepsilon Q h\|c\|_{2, \Omega_{i, j}}\left(\left\|\nabla\left(\tilde{R}_{h} c-c\right)\right\|_{\Omega_{i, j}}+\|\nabla \tilde{\rho}\|_{\Omega_{i, j}}\right) \\
& \leq \frac{\varepsilon D_{\min }}{2}\|\nabla \tilde{\rho}\|_{\Omega_{i, j}}^{2}+\varepsilon Q h^{2}\|c\|_{2, \Omega_{i, j}}^{2},
\end{aligned}
$$

which implies (23a).

Let $P_{h}$ denote the $L^{2}$-projection defined by $\left(w-P_{h} w, p\right)=0$ for $p \in$ $\mathcal{Q}_{0}\left(\Omega_{i, j}\right)$ with $\left\|w-P_{h} w\right\|_{\Omega_{i, j}} \leq Q h\|\nabla w\|_{\Omega_{i, j}}$, and rewrite (24a) into the following manner

$$
(\phi \tilde{\rho}, \tilde{\rho})_{\Omega_{i, j}}=(\phi \tilde{\rho}, \tilde{\rho}-w)_{\Omega_{i, j}}+(\phi \rho, w)_{\Omega_{i, j}} .
$$

Choosing $w=P_{h} \tilde{\rho}$ in (27), using Lemma 3.1 and the inverse estimate to yield

$$
\begin{aligned}
(\phi \tilde{\rho}, \tilde{\rho})_{\Omega_{i, j}} & =\left(\phi \tilde{\rho}, \tilde{\rho}-P_{h} \tilde{\rho}\right)_{\Omega_{i, j}}+\left(\phi \rho, P_{h} \tilde{\rho}\right)_{\Omega_{i, j}} \\
& \leq Q\|\tilde{\rho}\|_{\Omega_{i, j}}\left\|\tilde{\rho}-P_{h} \tilde{\rho}\right\|_{\Omega_{i, j}}+Q h^{2}\|c\|_{2, \Omega_{i, j}}\left\|P_{h} \tilde{\rho}\right\|_{\Omega_{i, j}} \\
& \leq Q h\|\tilde{\rho}\|_{\Omega_{i, j}}\|\nabla \tilde{\rho}\|_{\Omega_{i, j}}+Q h^{2}\|c\|_{2, \Omega_{i, j}}\|\tilde{\rho}\|_{\Omega_{i, j}} \\
& \leq \frac{\phi_{\min }}{2}\|\tilde{\rho}\|_{\Omega_{i, j}}^{2}+Q h^{4}\|c\|_{2, \Omega_{i, j}}^{2}
\end{aligned}
$$


After a simple calculation we obtain (23b).

We now turn to prove (23c). Differentiating (24a) and (24b) with respect to time $t$, we have

(a) $\left(\phi \tilde{\rho}_{t}, 1\right)_{\Omega_{i, j}}=\left(\phi_{t} \rho, 1\right)_{\Omega_{i, j}}+\left(\phi \rho_{t}, 1\right)_{\Omega_{i, j}}-\left(\phi_{t} \tilde{\rho}, 1\right)_{\Omega_{i, j}}$,

(b) $\varepsilon\left(D \nabla \tilde{\rho}_{t}, \nabla w\right)_{\Omega_{i, j}}=-\left(\boldsymbol{\eta}_{t}, \nabla w\right)_{\Omega_{i, j}}-\varepsilon\left(D_{t} \nabla \tilde{\rho}_{t}, \nabla w\right)_{\Omega_{i, j}}, \quad \forall w \in \tilde{V}_{h}$.

Rewriting (29a) as the following form

$$
\begin{aligned}
\varepsilon\left(D \nabla \tilde{\rho}_{t}, \nabla \tilde{\rho}_{t}\right)_{\Omega_{i, j}} & =\varepsilon\left(D \nabla \tilde{\rho}_{t}, \nabla\left(\tilde{\rho}_{t}-w\right)\right)_{\Omega_{i, j}} \\
& -\left(\boldsymbol{\eta}_{t}, \nabla w\right)_{\Omega_{i, j}}-\varepsilon\left(D_{t} \nabla \tilde{\rho}, \nabla w\right)_{\Omega_{i, j}} .
\end{aligned}
$$

Choosing $w=\left(\tilde{R}_{h} c-\tilde{C}_{h}\right)_{t}$ in (30) and arguing in the same way as done in the derivation of (23a) and (23b), we easily derive (23c) for $\tilde{\rho}_{t}$.

For later use we need to prove the following lemmas.

Lemma 3.4. For any element $\Omega_{i, j} \in \mathcal{T}_{h}$, the following expressions hold,

$$
\begin{aligned}
& \text { (a) }\left(\phi \tilde{e}^{n}, \tilde{e}^{n}-e^{n}\right)=\left\|\sqrt{\phi}\left(\tilde{e}^{n}-e^{n}\right)\right\|^{2}, \\
& \text { (b) }\left\|\sqrt{\phi} e^{n}\right\| \leq\left\|\sqrt{\phi} \tilde{e}^{n}\right\|, \\
& \text { (c) } \varepsilon\left\|\sqrt{D^{n}} \nabla \tilde{e}^{n}\right\|_{\Omega_{i, j}} \leq\left\|\frac{1}{\sqrt{D^{n}}} \xi^{n}\right\|_{\Omega_{i, j}}, \\
& \text { (d) }\left\|\sqrt{\phi}\left(\tilde{e}^{n}-e^{n}\right)\right\|_{\Omega_{i, j}} \leq Q\left\|\nabla \tilde{e}^{n}\right\|_{\Omega_{i, j}} h .
\end{aligned}
$$

Proof By (7) and (21), we have

$$
\begin{aligned}
& \text { (a) }\left(\phi\left(\tilde{e}^{n}-e^{n}\right), 1\right)_{\Omega_{i, j}}=0, \\
& \text { (b) }\left(\varepsilon D^{n} \nabla \tilde{e}^{n}+\boldsymbol{\xi}^{n}, \nabla w\right)_{\Omega_{i, j}}=0, \quad \forall w \in \tilde{V}_{h} .
\end{aligned}
$$

Since $e^{n}$ is a constant on $\Omega_{i, j},(31 \mathrm{a})$ and (31b) follow immediately from (32a). Taking $w=\tilde{e}^{n}$ in (32b) we obtain (31c). Finally (31d) follows from the following estimates,

$$
\begin{gathered}
\left(\phi\left(\tilde{e}^{n}-e^{n}\right), \tilde{e}^{n}-e^{n}\right)_{\Omega_{i, j}}=\left(\phi\left(\tilde{e}^{n}-e^{n}\right), \tilde{e}^{n}-P_{h} \tilde{e}^{n}\right)_{\Omega_{i, j}} \\
\leq\left\|\sqrt{\phi}\left(\tilde{e}^{n}-e^{n}\right)\right\|_{\Omega_{i, j}}\left\|\tilde{e}^{n}-P_{h} \tilde{e}^{n}\right\|_{\Omega_{i, j}} \\
\leq Q h\left\|\nabla \tilde{e}^{n}\right\|_{\Omega_{i, j}}\left\|\sqrt{\phi}\left(\tilde{e}^{n}-e^{n}\right)\right\|_{\Omega_{i, j}} .
\end{gathered}
$$


Lemma 3.5. Let $e^{n}$ satisfy

$$
\varepsilon\left(e^{n}, \nabla \cdot \boldsymbol{\chi}_{h}\right)-\left(D_{n}^{-1} \boldsymbol{\xi}^{n}, \boldsymbol{\chi}_{h}\right)=\left(D_{n}^{-1} \boldsymbol{\eta}^{n}, \boldsymbol{\chi}_{h}\right)-\varepsilon\left(\rho^{n}, \nabla \cdot \boldsymbol{\chi}_{h}\right) .
$$

Then there exist a function $\zeta^{n} \in H^{1}(\Omega)$ and some constant $Q$ independent of $h, n$ and $\varepsilon$ such that

$$
\begin{gathered}
\left\|\zeta^{n}\right\|_{1} \leq Q\left\|e^{n}\right\|, \\
\left\|\zeta^{n}-e^{n}\right\| \leq Q h\left(\left\|e^{n}\right\|+h\|c\|_{L^{\infty}\left(H^{2}\right)}\right) .
\end{gathered}
$$

Proof We define $W_{h} \subset H^{1}(\Omega)$ to be the space of continuous and piecewisebilinear functions determined by its nodal values over $\mathcal{T}_{h}$. Then we define a function $\zeta^{n} \in W_{h}$ by

$$
\left(\zeta^{n}-e^{n}, \omega\right)+\left(D^{n} \nabla \zeta^{n}, \nabla \omega\right)+\lambda\left|\left(\varepsilon^{-2}\left\|\boldsymbol{\xi}^{n}\right\|^{2}+1, \omega\right)\right|=0, \quad \forall \omega \in W_{h},
$$

where $\lambda$ is an undetermined positive constant independent of $n$ and $\varepsilon$.

We choose $\omega=\zeta^{n}$ in (37) and combine $D \geq D_{\min }>0$ with $\lambda \mid\left(\varepsilon^{-2}\left\|\boldsymbol{\xi}^{n}\right\|^{2}+\right.$ $\left.1, \zeta^{n}\right) \mid \geq 0$ to yield

$$
\left\|\zeta^{n}\right\|^{2}+D_{\min }\left\|\nabla \zeta^{n}\right\|^{2} \leq\left\|e^{n}\right\|\left\|\zeta^{n}\right\| .
$$

Thus we have (35). Moreover, (38) also implies the existence and uniqueness of $\zeta^{n}$.

Let $\varphi \in H^{2}(\Omega)$ solve the elliptic problem

$$
\begin{aligned}
(a) \varphi-\nabla \cdot\left(D^{n} \nabla \varphi\right) & =\zeta^{n}-e^{n} \text { in } \Omega, \\
(b)-D^{n} \nabla \varphi \cdot \mathbf{n} & =0 \text { on } \partial \Omega,
\end{aligned}
$$

so that

$$
\|\varphi\|_{2} \leq Q\left\|\zeta^{n}-e^{n}\right\|
$$


Choosing $\omega=\tilde{R}_{h} \varphi \in W_{h}$ in (37) and using (40) to derive

$$
\begin{aligned}
\| \zeta^{n}- & e^{n} \|^{2}=\left(\zeta^{n}-e^{n}, \varphi-\nabla \cdot\left(D^{n} \nabla \varphi\right)\right) \\
= & \left(\zeta^{n}-e^{n}, \varphi\right)-\left(\zeta^{n}-e^{n}, \nabla \cdot\left(D^{n} \nabla \varphi\right)\right) \\
= & \left(\zeta^{n}-e^{n}, \varphi-\tilde{R}_{h} \varphi\right)+\left(\zeta^{n}-e^{n}, \tilde{R}_{h} \varphi\right) \\
& -\left(\zeta^{n}, \nabla \cdot\left(D^{n} \nabla \varphi\right)\right)+\left(e^{n}, \nabla \cdot\left(D^{n} \nabla \varphi\right)\right) \\
= & \left(\zeta^{n}-e^{n}, \varphi-\tilde{R}_{h} \varphi\right)+\left(\zeta^{n}-e^{n}, \tilde{R}_{h} \varphi\right) \\
& +\left(\nabla \zeta^{n}, D^{n} \nabla \varphi\right)+\left(e^{n}, \nabla \cdot\left(D^{n} \nabla \varphi\right)\right) \\
= & \left(\zeta^{n}-e^{n}, \varphi-\tilde{R}_{h} \varphi\right)-\left(D^{n} \nabla \zeta^{n}, \nabla \tilde{R}_{h} \varphi\right)-\lambda\left|\left(\varepsilon^{-2}\left\|\boldsymbol{\xi}^{n}\right\|^{2}+1, \tilde{R}_{h} \varphi\right)\right| \\
& +\left(D^{n} \nabla \zeta^{n}, \nabla \varphi\right)+\left(e^{n}, \nabla \cdot\left(D^{n} \nabla \varphi\right)\right) \\
= & \left(\zeta^{n}-e^{n}, \varphi-\tilde{R}_{h} \varphi\right)+\left(D^{n} \nabla \zeta^{n}, \nabla\left(\varphi-\tilde{R}_{h} \varphi\right)\right) \\
& -\lambda\left|\left(\varepsilon^{-2}\left\|\boldsymbol{\xi}^{n}\right\|^{2}+1, \tilde{R}_{h} \varphi\right)\right|+\left(e^{n}, \nabla \cdot\left(D^{n} \nabla \varphi\right)\right) .
\end{aligned}
$$

We use (34) and the definition of $\Pi_{h}$ to obtain

$$
\begin{aligned}
\left(e^{n},\right. & \left.\nabla \cdot\left(D^{n} \nabla \varphi\right)\right)=\left(e^{n}, \nabla \cdot \Pi_{h}\left(D^{n} \nabla \varphi\right)\right) \\
= & \varepsilon^{-1}\left(D_{n}^{-1} \boldsymbol{\xi}^{n}, \Pi_{h}\left(D^{n} \nabla \varphi\right)\right) \\
& \quad+\varepsilon^{-1}\left(D_{n}^{-1} \boldsymbol{\eta}^{n}, \Pi_{h}\left(D^{n} \nabla \varphi\right)\right)-\left(\rho^{n}, \nabla \cdot \Pi_{h}\left(D^{n} \nabla \varphi\right)\right) .
\end{aligned}
$$

Taking (42) into (41) and combining (35) with (40), we have

$$
\begin{aligned}
& \left\|\zeta^{n}-e^{n}\right\|^{2}+\lambda\left|\left(1, \tilde{R}_{h} \varphi\right)\right| \varepsilon^{-2}\left\|\boldsymbol{\xi}^{n}\right\|^{2}+\lambda\left|\left(1, \tilde{R}_{h} \varphi\right)\right| \\
& =\left(\zeta^{n}-e^{n}, \varphi-\tilde{R}_{h} \varphi\right)+\left(D^{n} \nabla \zeta^{n}, \nabla\left(\varphi-\tilde{R}_{h} \varphi\right)\right)+\varepsilon^{-1}\left(D_{n}^{-1} \boldsymbol{\eta}^{n}, \Pi_{h}\left(D^{n} \nabla \varphi\right)\right) \\
& +\varepsilon^{-1}\left(D_{n}^{-1} \boldsymbol{\xi}^{n}, \Pi_{h}\left(D^{n} \nabla \varphi\right)\right)-\left(\rho^{n}, \nabla \cdot \Pi_{h}\left(D^{n} \nabla \varphi\right)\right) \\
& =\left(\zeta^{n}-e^{n}, \varphi-\tilde{R}_{h} \varphi\right)+\left(D^{n} \nabla \zeta^{n}, \nabla\left(\varphi-\tilde{R}_{h} \varphi\right)\right)+\varepsilon^{-1}\left(D_{n}^{-1} \boldsymbol{\eta}^{n}, \Pi_{h}\left(D^{n} \nabla \varphi\right)\right) \\
& +\varepsilon^{-1}\left(D_{n}^{-1} \boldsymbol{\xi}^{n}, \Pi_{h}\left(D^{n} \nabla \varphi\right)-D^{n} \nabla \varphi\right)+\varepsilon^{-1}\left(D_{n}^{-1} \boldsymbol{\xi}^{n}, D^{n} \nabla\left(\varphi-\tilde{R}_{h} \varphi\right)\right) \\
& +\varepsilon^{-1}\left(\boldsymbol{\xi}^{n}, \nabla \tilde{R}_{h} \varphi\right)-\left(\rho^{n}, \nabla \cdot \Pi_{h}\left(D^{n} \nabla \varphi\right)\right) \\
& \leq Q h^{2}\left\|\zeta^{n}-e^{n}\right\|\|\varphi\|_{2}+Q h\left\|\nabla \zeta^{n}\right\|\|\varphi\|_{2}+Q h^{2}\left\|c^{n}\right\|_{2}\|\varphi\|_{2}+Q h \varepsilon^{-1}\left\|\boldsymbol{\xi}^{n}\right\|\|\varphi\|_{2} \\
& +\varepsilon^{-1}\left\|\boldsymbol{\xi}^{n}\right\| \varphi\left\|_{2}+Q h^{2}\right\| c^{n}\left\|_{2}\right\| \varphi \|_{2} \\
& \leq Q h^{2}\left\|\zeta^{n}-e^{n}\right\|^{2}+Q h\left\|e^{n}\right\|\left\|\zeta^{n}-e^{n}\right\|+Q h \varepsilon^{-1}\left\|\boldsymbol{\xi}^{n}\right\|\left\|\zeta^{n}-e^{n}\right\| \\
& +\varepsilon^{-1}\left\|\boldsymbol{\xi}^{n}\right\|\left\|\zeta^{n}-e^{n}\right\|+Q h^{2}\|c\|_{L^{\infty}\left(H^{2}\right)}\left\|\zeta^{n}-e^{n}\right\| \\
& \leq Q h^{2}\left\|\zeta^{n}-e^{n}\right\|^{2}+\frac{7}{8}\left\|\zeta^{n}-e^{n}\right\|^{2}+\left(Q h^{2}+\frac{1}{2}\right) \varepsilon^{-2}\left\|\boldsymbol{\xi}^{n}\right\|^{2}+Q h^{2}\left\|e^{n}\right\|^{2}+Q h^{4}\|c\|_{L^{\infty}\left(H^{2}\right)}^{2} \text {. }
\end{aligned}
$$


According to the above estimates, we obtain the following inequality

$$
\begin{gathered}
\left(\frac{1}{8}-Q h^{2}\right)\left\|\zeta^{n}-e^{n}\right\|^{2}+\lambda\left|\left(1, \tilde{R}_{h} \varphi\right)\right| \varepsilon^{-2}\left\|\boldsymbol{\xi}^{n}\right\|^{2}+\lambda\left|\left(1, \tilde{R}_{h} \varphi\right)\right| \\
\leq Q h^{2}\left\|e^{n}\right\|^{2}+Q h^{4}\|c\|_{L^{\infty}\left(H^{2}\right)}^{2}+\left(Q h^{2}+\frac{1}{2}\right) \varepsilon^{-2}\left\|\boldsymbol{\xi}^{n}\right\|^{2}
\end{gathered}
$$

If $\left|\left(1, \tilde{R}_{h} \varphi\right)\right| \neq 0$, we use $(43)$ to get that

$$
\begin{aligned}
& \left(\frac{1}{8}-Q h^{2}\right)\left\|\zeta^{n}-e^{n}\right\|^{2}+\left(\lambda\left|\left(1, \tilde{R}_{h} \varphi\right)\right|-Q h^{2}-\frac{1}{2}\right) \varepsilon^{-2}\left\|\boldsymbol{\xi}^{n}\right\|^{2} \\
& \leq Q h^{2}\left\|e^{n}\right\|^{2}+Q h^{4}\|c\|_{L^{\infty}\left(H^{2}\right)}^{2} .
\end{aligned}
$$

Let $\lambda>\left(Q h^{2}+1\right) /\left|\left(1, \tilde{R}_{h} \varphi\right)\right|$ and $h$ is small enough, we easily obtain the estimate (36).

Otherwise assuming $\left|\left(1, \tilde{R}_{h} \varphi\right)\right|=0$, then $(1, \varphi)=\left(1, \varphi-\tilde{R}_{h} \varphi\right)$. Choosing $\omega=1$ in (39a), integrating and then applying the divergence theorem we have

$$
(1, \varphi)=\left(1, \zeta^{n}-e^{n}\right)
$$

Further choose $\omega=1$ in (37) and integrate to yield

$$
\left(1, \zeta^{n}-e^{n}\right)+\lambda \varepsilon^{-2}\left(a_{2}-a_{1}\right)\left(b_{2}-b_{1}\right)\left\|\boldsymbol{\xi}^{n}\right\|^{2}+\lambda\left(a_{2}-a_{1}\right)\left(b_{2}-b_{1}\right)=0,
$$

Take $\lambda=1$ and use (45) and (11) to have

$$
\begin{gathered}
\left(a_{2}-a_{1}\right)\left(b_{2}-b_{1}\right) \varepsilon^{-2}\left\|\boldsymbol{\xi}^{n}\right\|^{2}+\left(a_{2}-a_{1}\right)\left(b_{2}-b_{1}\right) \\
\quad=-\left(1, \zeta^{n}-e^{n}\right)=-\left(1, \varphi-\tilde{R}_{h} \varphi\right) \\
\quad \leq Q h^{2}\|\varphi\|_{2} \leq Q h^{2}+Q h^{2}\|\varphi\|_{2}^{2} .
\end{gathered}
$$

We choose sufficiently small $h$ such that $Q h^{2}<\left(a_{2}-a_{1}\right)\left(b_{2}-b_{1}\right)$ and combine (40) to get that

$$
\varepsilon^{-2}\left\|\boldsymbol{\xi}^{n}\right\|^{2} \leq Q h^{2}\left\|\zeta^{n}-e^{n}\right\|^{2} .
$$

Taking (48) and $\left|\left(1, \tilde{R}_{h} \varphi\right)\right|=0$ into (43), we have

$$
\left(\frac{1}{8}-Q h^{2}\right)\left\|\zeta^{n}-e^{n}\right\|^{2} \leq Q h^{2}\left\|e^{n}\right\|^{2}+Q h^{4}\|c\|_{L^{\infty}\left(H^{2}\right)}^{2}+Q h^{2}\left\|\zeta^{n}-e^{n}\right\|^{2} .
$$

A simple calculation on (49) leads the estimate (36) for sufficiently small $h$. 
Lemma 3.6. If $c \in L^{\infty}\left(0, T ; H^{3}(\Omega)\right)$, then the following estimate holds

$$
\left|-\varepsilon \Delta t^{n}\left(\rho^{n}, \nabla \cdot \boldsymbol{\xi}^{n}\right)\right| \leq \frac{\alpha_{0}}{4} \Delta t^{n}\left\|\boldsymbol{\xi}^{n}\right\|^{2}+\varepsilon Q \Delta t^{n} h^{4}\|c\|_{L^{\infty}\left(H^{3}\right)}^{2}
$$

Proof Let $\boldsymbol{\xi}=\left(\xi_{1}, \xi_{2}\right)^{T}$, then

$$
\begin{gathered}
-\varepsilon \Delta t^{n}\left(\rho^{n}, \nabla \cdot \boldsymbol{\xi}^{n}\right)=-\varepsilon \Delta t^{n}\left(\rho^{n}, \xi_{1 x}^{n}\right)-\varepsilon \Delta t^{n}\left(\rho^{n}, \xi_{2 y}^{n}\right) \\
=\varepsilon \Delta t^{n} \sum_{i, j}\left(\rho^{n}, \xi_{1 x}^{n}\right)_{\Omega_{i, j}}-\varepsilon \Delta t^{n} \sum_{i, j}\left(\rho^{n}, \xi_{2 y}^{n}\right)_{\Omega_{i, j}} .
\end{gathered}
$$

We mainly focus on the first term on the right side of (51), because the second one can be bounded by symmetry. By the expansion (13), we note that

$$
\begin{aligned}
\left(\rho^{n}, \xi_{1 x}^{n}\right)_{\Omega_{i, j}}= & -\int_{\Omega_{i, j}} \int_{x_{i-\frac{1}{2}}}^{x} c_{\alpha}\left(\alpha, y, t^{n}\right) d \alpha \xi_{1 x}\left(x, y, t^{n}\right) d x d y \\
& -\int_{\Omega_{i, j}} \int_{y_{j-\frac{1}{2}}} c_{\beta}\left(x, \beta, t^{n}\right) d \beta \xi_{1 x}\left(x, y, t^{n}\right) d x d y \\
& -\int_{\Omega_{i, j}} \int_{x_{i-\frac{1}{2}}}^{x} \int_{y_{j-\frac{1}{2}}}^{y} c_{\alpha \beta}\left(\alpha, \beta, t^{n}\right) d \alpha d \beta \xi_{1 x}\left(x, y, t^{n}\right) d x d y .
\end{aligned}
$$

We estimate the third term on the right side of (52) by

$$
\begin{aligned}
& \left|-\int_{\Omega_{i, j}} \int_{x_{i-\frac{1}{2}}}^{x} \int_{y_{j-\frac{1}{2}}}^{y} c_{\alpha \beta}\left(\alpha, \beta, t^{n}\right) d \alpha d \beta \xi_{1 x}\left(x, y, t^{n}\right) d x d y\right| \\
& =\left|\int_{\Omega_{i, j}} \int_{x_{i-\frac{1}{2}}}^{x} \int_{y_{j-\frac{1}{2}}}^{y} \int_{x_{i-\frac{1}{2}}}^{\alpha} c_{\gamma \gamma \beta}\left(\gamma, \beta, t^{n}\right) d \gamma d \alpha d \beta \xi_{1 x}\left(x, y, t^{n}\right) d x d y\right| \\
& \leq Q h^{3}\left\|\xi_{1 x}^{n}\right\|_{\Omega_{i, j}}\left\|c^{n}\right\|_{3, \Omega_{i, j}} \leq Q h^{2}\left\|\xi_{1}^{n}\right\|_{\Omega_{i, j}}\left\|c^{n}\right\|_{3, \Omega_{i, j}} .
\end{aligned}
$$

For the first and second terms, we need only to bound one of them, since 
the other is estimated similarly. The first term is

$$
\begin{aligned}
& -\int_{\Omega_{i, j}} \int_{x_{i-\frac{1}{2}}}^{x} c_{\alpha}\left(\alpha, y, t^{n}\right) d \alpha \xi_{1 x}\left(x, y, t^{n}\right) d x d y \\
& =\int_{\Omega_{i, j}} \int_{x_{i-\frac{1}{2}}}^{x} \int_{x_{i-\frac{1}{2}}}^{\alpha} c_{\beta \beta}\left(\beta, y, t^{n}\right) d \beta d \alpha \xi_{1 x}\left(x, y, t^{n}\right) d x d y \\
& =\int_{\Omega_{i, j}} \int_{x_{i-\frac{1}{3}}}^{\int_{x_{i-\frac{1}{2}}}} \int_{x_{i-\frac{1}{2}}}^{\beta} c_{\gamma \gamma \gamma}\left(\gamma, y, t^{n}\right) d \gamma d \beta d \alpha \xi_{1 x}\left(x, y, t^{n}\right) d x d y \\
& \quad+\int_{\Omega_{i, j}} \int_{x_{i-\frac{1}{2}}}^{\int_{x_{i-\frac{1}{2}}}^{\alpha}} c_{x x}\left(x_{i-\frac{1}{2}}, y, t^{n}\right) d \beta d \alpha \xi_{1 x}\left(x, y, t^{n}\right) d x d y \\
& \leq Q h^{3}\left\|\xi_{1 x}^{n}\right\|_{\Omega_{i, j}}\left\|c^{n}\right\|_{3, \Omega_{i, j}}+\int_{\Omega_{i, j}} \int_{x_{i-\frac{1}{2}}}^{x} \int_{x_{i-\frac{1}{2}}}^{\alpha} c_{x x}\left(x_{i-\frac{1}{2}}, y, t^{n}\right) d \beta d \alpha \xi_{1 x}\left(x, y, t^{n}\right) d x d y \\
& \leq Q h^{2}\left\|\xi_{1}^{n}\right\|_{\Omega_{i, j}}\left\|c^{n}\right\|_{3, \Omega_{i, j}}+\int_{\Omega_{i, j}} c_{x x}\left(x_{i-\frac{1}{2}}, y, t^{n}\right)\left(x-x_{i-\frac{1}{2}}\right)^{2} \xi_{1 x}\left(x, y, t^{n}\right) d x d y
\end{aligned}
$$

To obtain an optimal-order error estimate for the second term on the right side of (54), we need to transform the integral area $\Omega_{i, j}$ into the domain $\Omega$. By the periodic boundary condition, we have

$$
\begin{aligned}
& \left|\sum_{i, j} \int_{\Omega_{i, j}} c_{x x}\left(x_{i-\frac{1}{2}}, y, t^{n}\right)\left(x-x_{i-\frac{1}{2}}\right)^{2} \xi_{1 x}\left(x, y, t^{n}\right) d x d y\right| \\
& =\frac{(\Delta x)^{2}}{24}\left|\int_{c}^{d} \sum_{i=1}^{I} c_{x x}\left(x_{i-\frac{1}{2}}, y, t^{n}\right)\left(\xi_{1}\left(x_{i}, y, t^{n}\right)-\xi_{1}\left(x_{i-1}, y, t^{n}\right)\right) d y\right| \\
& \leq \frac{(\Delta x)^{2}}{24}\left|\int_{c}^{d}\left(c_{x x}\left(x_{I-\frac{1}{2}}, y, t^{n}\right) \xi_{1}\left(x_{I}, y, t^{n}\right)-c_{x x}\left(x_{\frac{1}{2}}, y, t^{n}\right) \xi_{1}\left(x_{0}, y, t^{n}\right)\right) d y\right| \\
& \left.\quad-\sum_{i=1}^{I-1}\left(c_{x x}\left(x_{i+\frac{1}{2}}, y, t^{n}\right)-c_{x x}\left(x_{i-\frac{1}{2}}, y, t^{n}\right)\right) \xi_{1}\left(x_{i}, y, t^{n}\right)\right\} d y \mid \\
& \leq Q h^{2}\left|\int_{c}^{d} \int_{x_{\frac{1}{2}}}^{x_{I-\frac{1}{2}}} c_{x x x} \xi_{1}\left(a, y, t^{n}\right) d x d y\right| \\
& \quad+Q h^{2}\left|\int_{c}^{d} \sum_{i=1}^{I-1} \int_{x_{i-\frac{1}{2}}}^{x_{i+\frac{1}{2}}} c_{x x x}\left(x, y, t^{n}\right) \xi_{1}\left(x_{i}, y, t^{n}\right) d x d y\right| \\
& \leq Q h^{2}\left\|c^{n}\right\|_{3}\left\|\xi_{1}^{n}\right\| .
\end{aligned}
$$

Combining these estimates, we complete the proof. 


\section{4. $\varepsilon$-Uniform Error Estimates}

In this section, we shall conduct $\varepsilon$-uniform error estimates for the characteristicsmixed finite element scheme (6) and the postprocessing scheme (7).

Theorem 4.1. Let $c \in L^{\infty}\left(0, T ; H^{2}(\Omega)\right) \cap H^{2}\left(0, T ; H^{2}(\Omega)\right)$ and $\left(\mathbf{z}_{h}, c_{h}\right)$ be the solutions to (5) and (6) respectively. And let $\Delta t \geq K^{\prime} h^{\frac{3}{2}}$. Then there exists some constant $Q$ independent of $h, \Delta t$ and $\varepsilon$ such that the following uniform optimal-order error estimate holds

$$
\begin{aligned}
& \left\|c-c_{h}\right\|_{L^{\infty}\left(L^{2}\right)}+\left.\left\|\mathbf{\| z}-\mathbf{z}_{h}\right\|\right|_{L^{2}\left(L_{\varepsilon}^{2}\right)} \leq Q\left\{h\left(\|c\|_{L^{\infty}\left(H^{2}\right)}+\|c\|_{H^{1}\left(H^{2}\right)}\right)\right. \\
& \left.\quad+\Delta t\left(\left\|(\nabla \cdot \mathbf{z})_{\tau}\right\|_{L^{2}\left(L^{2}\right)}+\|\nabla \cdot \mathbf{z}\|_{L^{2}\left(L^{2}\right)}+\left\|c_{\tau}\right\|_{L^{2}\left(L^{2}\right)}+\|c\|_{L^{2}\left(H^{1}\right)}\right)\right\} .
\end{aligned}
$$

Proof By (5), (6), (22) and the properties of $\Pi_{h}$, we obtain the error equation, for $\forall w_{h} \in V_{h}$ and $\forall \chi_{h} \in \mathbf{H}_{h}$,

$$
\begin{aligned}
& \text { (a) }\left(\phi^{n} e^{n}, w_{h}\right)-\left(\phi^{n-1} \tilde{e}^{n-1}, \hat{w}_{h}^{n-1,+}\right)+\left(\nabla \cdot \boldsymbol{\xi}^{n}, w_{h}\right) \Delta t^{n}+\int_{t^{n-1}}^{t^{n}}\left(f_{2} \hat{e}, \hat{w}_{h}\right) d t \\
& =\int_{t^{n-1}}^{t^{n}}\left(f_{2}\left(\hat{R_{h}} c-c\right), \hat{w}_{h}\right) d t+\int_{t^{n-1}}^{t^{n}}\left(\nabla \cdot[(\hat{\mathbf{u}}-\mathbf{u}) c], \hat{w}_{h}\right) d t \\
& +\left(\phi^{n-1} \tilde{\rho}^{n-1}, \hat{w}_{h}^{n-1,+}\right)-\left(\phi^{n} \tilde{\rho}^{n}, w_{h}\right)+\int_{t^{n-1}}^{t^{n}}\left[\left(\nabla \cdot \mathbf{z}^{n}, w_{h}\right)-\left(\nabla \cdot \mathbf{z}, \hat{w}_{h}\right)\right] d t, \\
& \text { (b) } \varepsilon\left(e^{n}, \nabla \cdot \boldsymbol{\chi}_{h}\right)-\left(D_{n}^{-1} \boldsymbol{\xi}^{n}, \boldsymbol{\chi}_{h}\right)=\left(D_{n}^{-1} \boldsymbol{\eta}^{n}, \boldsymbol{\chi}_{h}\right)-\varepsilon\left(\rho^{n}, \nabla \cdot \boldsymbol{\chi}_{h}\right) \text {. }
\end{aligned}
$$

Multiplying (57a) by $\varepsilon$ and (57b) by $\Delta t$, taking $w_{h}=e^{n}, \boldsymbol{\chi}_{h}=\boldsymbol{\xi}^{n}$ and adding, we get

$$
\begin{aligned}
& \varepsilon\left(\phi^{n} e^{n}, e^{n}\right)-\varepsilon\left(\phi^{n-1} \tilde{e}^{n-1}, \hat{e}^{n-1,+}\right)+\Delta t\left(D_{n}^{-1} \boldsymbol{\xi}^{n}, \boldsymbol{\xi}^{n}\right)+\varepsilon \int_{t^{n-1}}^{t^{n}}\left(f_{2} \hat{e}, \hat{e}\right) d t \\
& \quad=\varepsilon \int_{t^{n-1}}^{t^{n}}\left(f_{2}\left(\hat{R_{h}} c-c\right), \hat{e}\right) d t+\varepsilon \int_{t^{n-1}}^{t^{n}}(\nabla \cdot[(\hat{\mathbf{u}}-\mathbf{u}) c], \hat{e}) d t \\
& \quad+\varepsilon\left(\phi^{n-1} \tilde{\rho}^{n-1}, \hat{e}^{n-1,+}\right)-\varepsilon\left(\phi^{n} \tilde{\rho}^{n}, e^{n}\right)+\varepsilon \int_{t^{n-1}}^{t^{n}}\left[\left(\nabla \cdot \mathbf{z}^{n}, e^{n}\right)-(\nabla \cdot \mathbf{z}, \hat{e})\right] d t \\
& \quad-\Delta t\left(D_{n}^{-1} \boldsymbol{\eta}^{n}, \boldsymbol{\xi}^{n}\right)-\varepsilon \Delta t\left(\rho^{n}, \nabla \cdot \boldsymbol{\xi}^{n}\right) .
\end{aligned}
$$

Noting that $\hat{e}^{n-1,+}$ is a constant on $\Omega_{i, j}$ and using (32a) we estimate the second term on the left-hand side of (58) as follows

$$
\begin{aligned}
& \varepsilon\left(\phi^{n-1} \tilde{e}^{n-1}, \hat{e}^{n-1,+}\right)=\varepsilon\left(\phi^{n-1} e^{n-1}, \hat{e}^{n-1,+}\right) \\
& \quad=\varepsilon\left(\phi^{n-1} e^{n-1}, e^{n-1}\right)-\varepsilon\left(\phi^{n-1} e^{n-1}, e^{n-1}-\hat{e}^{n-1,+}\right) \\
& \quad \leq \frac{\varepsilon}{2}\left(\phi^{n-1} e^{n-1}, e^{n-1}\right)+\frac{\varepsilon}{2}\left(\phi^{n-1} \hat{e}^{n-1,+}, \hat{e}^{n-1,+}\right) \\
& \quad \leq \frac{\varepsilon}{2}\left(\phi^{n-1} e^{n-1}, e^{n-1}\right)+\frac{\varepsilon}{2}\left(\phi^{n} e^{n}, e^{n}\right)+\varepsilon K\left\|e^{n}\right\|^{2} \Delta t .
\end{aligned}
$$


Here the inequality $\left(\phi^{n-1} \hat{e}^{n-1,+}, \hat{e}^{n-1,+}\right) \leq\left(\phi^{n} e^{n}, e^{n}\right)+\varepsilon K\left\|e^{n}\right\|^{2} \Delta t$ proved in Arbogast \& Wheeler [1] is used. Therefore we have

$$
\begin{aligned}
& \varepsilon\left(\phi^{n} e^{n}, e^{n}\right)-\varepsilon\left(\phi^{n-1} \tilde{e}^{n-1}, \hat{e}^{n-1,+}\right) \\
& \quad \geq \frac{\varepsilon}{2}\left(\phi^{n} e^{n}, e^{n}\right)-\frac{\varepsilon}{2}\left(\phi^{n-1} e^{n-1}, e^{n-1}\right)-\varepsilon K\left\|e^{n}\right\|^{2} \Delta t^{n} .
\end{aligned}
$$

An application of (60) and a simple calculation for (58) lead to

$$
\begin{aligned}
& \frac{\varepsilon}{2}\left[\left(\phi^{n} e^{n}, e^{n}\right)-\left(\phi^{n-1} e^{n-1}, e^{n-1}\right)\right]+\Delta t\left\|D_{n}^{-1} \boldsymbol{\xi}^{n}\right\|^{2}+\varepsilon \int_{t^{n-1}}^{t^{n}}\left(f_{2} \hat{e}, \hat{e}\right) d t \\
& \leq \varepsilon \int_{t^{n-1}}^{t^{n}}\left(f_{2}\left(\hat{R_{h} c}-c\right), \hat{e}\right) d t+\varepsilon \int_{t^{n-1}}^{t^{n}}(\nabla \cdot[(\hat{\mathbf{u}}-\mathbf{u}) c], \hat{e}) d t \\
& \quad+\varepsilon\left(\phi^{n-1} \tilde{\rho}^{n-1}, \hat{e}^{n-1,+}\right)-\varepsilon\left(\phi^{n} \tilde{\rho}^{n}, e^{n}\right)+\varepsilon \int_{t^{n-1}}^{t^{n}}\left[\left(\nabla \cdot \mathbf{z}^{n}, e^{n}\right)-(\nabla \cdot \mathbf{z}, \hat{e})\right] d t \\
& \quad-\Delta t^{n}\left(D_{n}^{-1} \boldsymbol{\eta}^{n}, \boldsymbol{\xi}^{n}\right)-\varepsilon \Delta t^{n}\left(\rho^{n}, \nabla \cdot \boldsymbol{\xi}^{n}\right)+\varepsilon K \|^{2} \Delta t,
\end{aligned}
$$

in which eight terms on the right-hand side will be bounded term by term as follows.

Compared to the error equation (6.2) in Arbogast \& Wheeler [1] we notice that the modification in (57) or (61) in this article is the replacement of the mixed elliptic projection by the interpolation operators defined in Section 3. This leads the additional seventh term, and makes the estimates of the third and the fourth terms become more difficult. Hence we mainly focus on the proof in details for these three terms and only quote the corresponding estimates from Arbogast \& Wheeler [1] for the remaining five terms.

We estimate the first term from Lemma 2 and the inequality (6.14) in Arbogast \& Wheeler [1],

$$
\begin{aligned}
& \varepsilon \int_{t^{n-1}}^{t^{n}}\left(f_{2}\left(\hat{R_{h}} c-c\right), \hat{e}\right) d t \\
& \quad \leq \varepsilon Q \Delta t^{n}\left\|e^{n}\right\|^{2}+\varepsilon Q \Delta t^{n} h^{4}\|c\|_{L^{\infty}\left(H^{2}\right)}^{2}+\varepsilon Q\left(\Delta t^{n}\right)^{2} \int_{t^{n-1}}^{t^{n}}\left\|c_{\tau}\right\|^{2} d t
\end{aligned}
$$

and the second term from Lemma 3 and the inequality (6.23) of Arbogast \& Wheeler [1],

$$
-\varepsilon \int_{t^{n-1}}^{t^{n}}(\nabla \cdot[(\hat{\mathbf{u}}-\mathbf{u}) c], \hat{e}) d t \leq \varepsilon Q(\Delta t)^{2} \int_{t^{n-1}}^{t^{n}}\|c\|_{1}^{2} d t+\varepsilon Q\left\|e^{n}\right\|^{2} \Delta t .
$$


The fifth term is estimated with Lemma 2 and 3 of Arbogast \& Wheeler [1],

$$
\begin{aligned}
& \left|\varepsilon \int_{t^{n-1}}^{t^{n}}\left[\left(\nabla \cdot \mathbf{z}^{n}, e^{n}\right)-(\nabla \cdot \mathbf{z}, \hat{e})\right] d t\right| \\
& \quad \leq \varepsilon Q \Delta t\left\{\int_{t^{n-1}}^{t^{n}}\left(\left\|(\nabla \cdot \mathbf{z})_{\tau}\right\|^{2}+\|\nabla \cdot \mathbf{z}\|^{2}\right) d t \Delta t+\left\|e^{n}\right\|^{2}\right\} .
\end{aligned}
$$

By the approximation property of $\Pi_{h}$ and the assumption $\mathbf{z}=-\varepsilon D \nabla c$, we bound the sixth term as follows

$$
\begin{aligned}
& \Delta t\left(D_{n}^{-1} \boldsymbol{\eta}^{n}, \boldsymbol{\xi}^{n}\right) \mid \leq Q \Delta t h\left\|\mathbf{z}^{n}\right\|_{1}\left\|\boldsymbol{\xi}^{n}\right\| \\
& \quad \leq Q \varepsilon \Delta t^{n} h^{2}\|c\|_{L^{\infty}\left(H^{2}\right)}^{2}+\frac{\alpha_{0} \varepsilon}{4} \Delta t\left\|\boldsymbol{\xi}^{n}\right\|^{2} .
\end{aligned}
$$

The remaining is to estimate those three terms. We notice that if the seventh term is bounded directly by (10) and the inverse inequality, It would lead a suboptimal-order error estimate. Hence we shall use the super-approximation property of $R_{h}$ presented in Lemma 3.1 instead of (10) to derive an optimalorder error estimate,

$$
\begin{gathered}
\left|\varepsilon \Delta t\left(\rho^{n}, \nabla \cdot \boldsymbol{\xi}^{n}\right)\right| \leq Q \varepsilon \Delta t^{n} h^{2}\left\|c^{n}\right\|_{2}\left\|\nabla \cdot \boldsymbol{\xi}^{n}\right\| \\
\leq Q \varepsilon \Delta t h^{2}\left\|c^{n}\right\|_{L^{\infty}\left(H^{2}\right)}^{2}+\frac{\alpha_{0} \varepsilon}{4} \Delta t\left\|\boldsymbol{\xi}^{n}\right\|^{2} .
\end{gathered}
$$

To bound the third and forth terms, we shall use Lemma 3.5 to find an $H^{1}(\Omega)$-function to replace $e^{n}$ and carry out an $H^{-1}$-dual argument. Firstly, we decompose them as the follows

$$
\begin{aligned}
& \varepsilon\left|\left(\phi^{n-1} \tilde{\rho}^{n-1}, \hat{e}^{n-1,+}\right)-\left(\phi^{n} \tilde{\rho}^{n}, e^{n}\right)\right| \\
& \quad=\left|\varepsilon\left((\check{\phi} \tilde{\rho})^{n-1,+}-(\phi \tilde{\rho})^{n-1}, e^{n}\right)+\varepsilon\left((\check{\phi} \tilde{\rho})^{n-1,+}, e^{n} J_{n}^{\prime}\right)-\varepsilon \int_{t^{n-1}}^{t^{n}}\left((\phi \tilde{\rho})_{t}, e^{n}\right) d t\right| \\
& \quad \leq \varepsilon\left|\left((\check{\phi} \tilde{\rho})^{n-1,+}-(\phi \tilde{\rho})^{n-1}, e^{n}\right)\right|+\varepsilon Q \Delta t\left\|\tilde{\rho}^{n-1}\right\|\left\|e^{n}\right\|+\varepsilon\left|\int_{t^{n-1}}^{t^{n}}\left((\phi \tilde{\rho})_{t}, e^{n}\right) d t\right| .
\end{aligned}
$$

Here we used $\left(\psi_{1}, \hat{\psi}_{2}\right)=\left(\check{\psi}_{1}, \psi_{2} J_{n}\right)=\left(\check{\psi}_{1}, \psi_{2}\right)+\left(\check{\psi}_{1}, \psi_{2} J_{n}^{\prime}\right)$ for some $J_{n}(\mathbf{x}, t)=$ $1+J_{n}^{\prime}(\mathbf{x}, t)$ such that $\left|J_{n}^{\prime}(\mathbf{x}, t)\right| \leq K\left(t^{n}-t\right)$. (See Lemma 2 in Arbogast \& Wheeler [1]).

Let $\zeta$ be an $H^{1}$-function defined in Lemma 3.5. Then the first term in 
(67) is changed to

$$
\begin{aligned}
& \left((\check{\phi} \tilde{\rho})^{n-1,+}-(\phi \tilde{\rho})^{n-1}, e^{n}\right) \\
& \quad=\left((\check{\phi} \tilde{\rho})^{n-1,+}-(\phi \tilde{\rho})^{n-1}, \zeta^{n}\right)+\left((\check{\phi} \tilde{\rho})^{n-1,+}-(\phi \tilde{\rho})^{n-1}, e^{n}-\zeta^{n}\right) \\
& \quad \leq Q\left\|(\check{\phi} \tilde{\rho})^{n-1,+}-(\phi \tilde{\rho})^{n-1}\right\|_{-1}\left\|e^{n}\right\|_{-1}+Q h\left\|e^{n}\right\|_{-1}\left\|(\check{\phi} \tilde{\rho})^{n-1,+}-(\phi \tilde{\rho})^{n-1}\right\| .
\end{aligned}
$$

Following Chen et al. [3], Douglas \& Russell [6], we have

$$
\begin{gathered}
\left\|(\check{\phi} \tilde{\rho})^{n-1,+}-(\phi \tilde{\rho})^{n-1}\right\|_{-1} \leq Q\left\|\tilde{\rho}^{n-1}\right\| \Delta t^{n}, \\
\left\|(\check{\phi} \tilde{\rho})^{n-1,+}-(\phi \tilde{\rho})^{n-1}\right\| \leq Q\left\|\tilde{\rho}^{n-1}\right\| .
\end{gathered}
$$

Taking (69), (70) into (68) and using the assumption $\Delta t \geq K^{\prime} h^{\frac{3}{2}}$ to yield

$$
\begin{aligned}
& \left((\check{\phi} \tilde{\rho})^{n-1,+}-(\phi \tilde{\rho})^{n-1}, e^{n}\right) \\
& \quad \leq Q \Delta t\left\|\tilde{\rho}^{n-1}\right\|\left\|e^{n}\right\|_{-1}+Q h\left\|\tilde{\rho}^{n-1}\right\|\left\|e^{n}\right\|_{-1} \\
& \quad \leq Q \Delta t^{n} h^{2}\left\|e^{n}\right\|\|c\|_{L^{\infty}\left(H^{2}\right)}+Q \Delta t^{n} h^{\frac{3}{2}}\left\|e^{n}\right\|\|c\|_{L^{\infty}\left(H^{2}\right)} \\
& \quad \leq Q \Delta t\left\|e^{n}\right\|^{2}+Q \Delta t^{n} h^{3}\|c\|_{L^{\infty}\left(H^{2}\right)}^{2} .
\end{aligned}
$$

Further we have

$$
\begin{gathered}
\varepsilon\left|\int_{t^{n-1}}^{t^{n}}\left((\phi \tilde{\rho})_{t}, e^{n}\right) d t\right| \leq \varepsilon Q \int_{t^{n-1}}^{t^{n}}\left(\left\|\tilde{\rho}_{t}\right\|+\|\tilde{\rho}\|\right)\left\|e^{n}\right\| d t \\
\leq \varepsilon Q h^{4} \int_{t^{n-1}}^{t^{n}}\left(\left\|c_{t}\right\|_{2}^{2}+\|c\|_{2}^{2}\right) d t+\varepsilon Q \Delta t\left\|e^{n}\right\|^{2} .
\end{gathered}
$$

Finally we take (71) and (72) into (67) to conclude the estimates for the third and fourth terms.

$$
\begin{gathered}
\left.\varepsilon \mid\left(\phi^{n-1} \tilde{\rho}^{n-1}, \hat{e}^{n-1,+}\right)-\left(\phi^{n} \tilde{\rho}^{n}, e^{n}\right)\right) \mid \leq Q \Delta t\left\|e^{n}\right\|^{2} \\
\quad+Q \varepsilon h^{3}\left(\Delta t\|c\|_{L^{\infty}\left(H^{2}\right)}^{2}+\int_{t^{n-1}}^{t^{n}}\left(\left\|c_{t}\right\|_{2}^{2}+\|c\|_{2}^{2}\right) d t\right) .
\end{gathered}
$$

Collecting the estimates for these seven terms and noting that $f_{2} \geq 0$, we have

$$
\begin{aligned}
& \varepsilon\left(\left\|\sqrt{\phi^{n}} e^{n}\right\|^{2}-\left\|\sqrt{\phi^{n-1}} e^{n-1}\right\|^{2}\right)+\frac{\alpha_{0}}{2} \Delta t\left\|\boldsymbol{\xi}^{n}\right\|^{2} \\
& \quad \leq \varepsilon Q \Delta t^{n}\left\|e^{n}\right\|^{2}+\varepsilon Q \Delta t^{n} h^{2}\|c\|_{L^{\infty}\left(H^{2}\right)}^{2}+\varepsilon Q h^{2} \int_{t^{n-1}}^{t^{n}}\left(\left\|c_{t}\right\|_{2}^{2}+\|c\|_{2}^{2}\right) d t \\
& \quad+\varepsilon Q(\Delta t)^{2} \int_{t^{n-1}}^{t^{n}}\left(\left\|c_{\tau}\right\|^{2}+\|c\|_{1}^{2}+\left\|(\nabla \cdot \mathbf{z})_{\tau}\right\|^{2}+\|\nabla \cdot \mathbf{z}\|^{2}\right) d t .
\end{aligned}
$$


Selecting the initial value $c_{h}(0)=R_{h} c(0)$ and adding, we have the following inequality by an application of Gronwall's Lemma

$$
\begin{aligned}
& \left\|e^{n}\right\|_{L^{\infty}\left(L^{2}\right)}+\mid\left\|\boldsymbol{\xi}^{n}\right\|_{L^{2}\left(L_{\varepsilon}^{2}\right)} \leq Q h\left(\|c\|_{L^{\infty}\left(H^{2}\right)}+\|c\|_{H^{1}\left(H^{2}\right)}\right) \\
& \quad+Q \Delta t^{n}\left(\left\|c_{\tau}\right\|_{L^{2}\left(L^{2}\right)}+\|c\|_{L^{2}\left(H^{1}\right)}+\left\|(\nabla \cdot \mathbf{z})_{\tau}\right\|_{L^{2}\left(L^{2}\right)}+\|\nabla \cdot \mathbf{z}\|_{L^{2}\left(L^{2}\right)}\right) .
\end{aligned}
$$

A combination of (75), (10) and (19) implies the conclusion of the theorem.

Now we turn to present $\varepsilon$-uniform error estimates for the postprocessing scheme (7).

Theorem 4.2. Let $(\mathbf{z}, c)$ be the solutions of $(5), c \in L^{\infty}\left(0, T ; H^{3}(\Omega)\right) \cap$ $H^{2}\left(0, T ; H^{2}(\Omega)\right)$ and $\tilde{c}_{h}$ is the solution of postprocessing scheme (7). Then there exist some constants $Q$ independent of $h, \Delta t$ and $\varepsilon$ such that the following uniform optimal-order error estimates hold for $\Delta t \geq K^{\prime} h^{\frac{3}{2}}$,

$$
\begin{aligned}
& \left\|c-\tilde{c}_{h}\right\|_{L^{\infty}\left(L^{2}\right)} \leq Q\left\{h^{\frac{3}{2}}\left(\|c\|_{L^{\infty}\left(H^{3}\right)}+\|c\|_{H^{1}\left(H^{2}\right)}\right)\right. \\
& \left.\quad+\Delta t\left(\left\|(\nabla \cdot \mathbf{z})_{\tau}\right\|_{L^{2}\left(L^{2}\right)}+\|\nabla \cdot \mathbf{z}\|_{L^{2}\left(L^{2}\right)}+\left\|c_{\tau}\right\|_{L^{2}\left(L^{2}\right)}+\|c\|_{L^{2}\left(H^{1}\right)}\right)\right\} .
\end{aligned}
$$

Proof We begin the proof by representing the left-hand side of the error equation (61) by $\tilde{e}$ and $e$. Indeed, by using Lemma 4 in Arbogast \& Wheeler [1] and the inequality $a(a-b) \geq \frac{1}{2}\left(a^{2}-b^{2}\right)$, we have

$$
\begin{aligned}
& \left(\phi^{n-1} \tilde{e}^{n-1}, \hat{e}^{n-1,+}\right)=\left(\phi^{n-1} \tilde{e}^{n-1}, \tilde{e}^{n-1}\right)-\left(\phi^{n-1} \tilde{e}^{n-1}, \tilde{e}^{n-1}-\hat{e}^{n-1,+}\right) \\
& \quad \leq \frac{1}{2}\left(\phi^{n-1} \tilde{e}^{n-1}, \tilde{e}^{n-1}\right)+\frac{1}{2}\left(\phi^{n-1} \hat{e}^{n-1,+}, \hat{e}^{n-1,+}\right) \\
& \leq \frac{1}{2}\left(\phi^{n-1} \tilde{e}^{n-1}, \tilde{e}^{n-1}\right)+\frac{1}{2}\left(\phi^{n} e^{n}, e^{n}\right)+K\left\|e^{n}\right\|^{2} \Delta t^{n}
\end{aligned}
$$

Then (32a) and (77) lead

$$
\begin{aligned}
& \varepsilon\left(\phi^{n} e^{n}, e^{n}\right)-\varepsilon\left(\phi^{n-1} \tilde{e}^{n-1}, \hat{e}^{n-1,+}\right) \\
& \quad \geq \frac{\varepsilon}{2}\left(\phi^{n} \tilde{e}^{n}, e^{n}\right)-\frac{\varepsilon}{2}\left(\phi^{n-1} \tilde{e}^{n-1}, \tilde{e}^{n-1}\right)-\varepsilon K\left\|e^{n}\right\|^{2} \Delta t^{n} \\
& \quad \geq \frac{\varepsilon}{2}\left[\left(\phi^{n} \tilde{e}^{n}, \tilde{e}^{n}\right)-\left(\phi^{n-1} \tilde{e}^{n-1}, \tilde{e}^{n-1}\right)\right]-\varepsilon K\left\|e^{n}\right\|^{2} \Delta t^{n}-\frac{\varepsilon}{2}\left(\phi^{n} \tilde{e}^{n}, \tilde{e}^{n}-e^{n}\right) .
\end{aligned}
$$

Using (31) to bound the last term of (78) to yield

$$
\begin{aligned}
\varepsilon\left(\phi^{n} e^{n}, e^{n}\right) & -\varepsilon\left(\phi^{n-1} \tilde{e}^{n-1}, \hat{e}^{n-1,+}\right) \\
& \geq \frac{\varepsilon}{2}\left[\left(\phi^{n} \tilde{e}^{n}, \tilde{e}^{n}\right)-\left(\phi^{n-1} \tilde{e}^{n-1}, \tilde{e}^{n-1}\right)\right]-\varepsilon K\left\|e^{n}\right\|^{2} \Delta t^{n}-K h^{2}\left\|\boldsymbol{\xi}^{n}\right\|^{2} .
\end{aligned}
$$


Hence the left-hand side of the error equation (61) has the following lower bound,

$$
\frac{\varepsilon}{2}\left[\left(\phi^{n} \tilde{e}^{n}, \tilde{e}^{n}\right)-\left(\phi^{n-1} \tilde{e}^{n-1}, \tilde{e}^{n-1}\right)\right]+\alpha_{0} \Delta t^{n}\left\|\boldsymbol{\xi}^{n}\right\|^{2}+\varepsilon \int_{t^{n-1}}^{t^{n}}\left(f_{2} \hat{e}, \hat{e}\right) d t .
$$

We now are back to the right-hand side of (61). Note that the convergence orders of the first five terms are high enough to satisfy (76), so it is unnecessary to re-estimate them term by term. Here we only pay our full attention to enhance the convergence order of the sixth and seventh terms.

For the sixth term we use (20) here instead of (19) and combine $\mathbf{z}=$ $-\varepsilon D \nabla c$ to obtain

$$
\begin{aligned}
& \left|\Delta t^{n}\left(D_{n}^{-1} \boldsymbol{\eta}^{n}, \boldsymbol{\xi}^{n}\right)\right| \leq Q \Delta t^{n} h^{2}\left\|\mathbf{z}^{n}\right\|_{2}\left\|\boldsymbol{\xi}^{n}\right\| \\
& \quad=Q \Delta t^{n} h^{2}\left\|-\varepsilon D^{n} \nabla c^{n}\right\|_{2}\left\|\boldsymbol{\xi}^{n}\right\| \\
& \quad \leq Q \varepsilon \Delta t^{n} h^{4}\|c\|_{L^{\infty}\left(H^{3}\right)}^{2}+\frac{\alpha_{0}}{4} \Delta t^{n}\left\|\boldsymbol{\xi}^{n}\right\|^{2} .
\end{aligned}
$$

We use Lemma 3.6 to derive a better estimate for the seventh term.

$$
\left|-\varepsilon \Delta t^{n}\left(\rho^{n}, \nabla \cdot \boldsymbol{\xi}^{n}\right)\right| \leq \frac{\alpha_{0}}{4} \Delta t^{n}\left\|\boldsymbol{\xi}^{n}\right\|^{2}+\varepsilon Q \Delta t^{n} h^{4}\|c\|_{L^{\infty}\left(H^{3}\right)}^{2} .
$$

Taking (81), (82) and the estimates for the first five terms done before into (80) we obtain

$$
\begin{aligned}
& \frac{\varepsilon}{2}\left\|\sqrt{\phi^{n}} \tilde{e}^{n}\right\|^{2}-\frac{\varepsilon}{2}\left\|\sqrt{\phi^{n-1}} \tilde{e}^{n-1}\right\|^{2}+\left(\frac{\alpha_{0}}{2} \Delta t^{n}-K h^{2}\right)\left\|\boldsymbol{\xi}^{n}\right\|^{2} \\
& \leq \varepsilon Q\left\{\Delta t^{n} h^{\frac{3}{2}}\|c\|_{L^{\infty}\left(H^{3}\right)}^{2}+h^{4} \int_{t^{n-1}}^{t^{n}}\left(\left\|c_{t}\right\|_{2}^{2}+\|c\|_{2}^{2}\right) d t+\Delta t^{n}\left\|e^{n}\right\|^{2}\right. \\
& \left.\quad+\left(\Delta t^{n}\right)^{2} \int_{t^{n-1}}^{t^{n}}\left(\left\|(\nabla \cdot \mathbf{z})_{\tau}\right\|^{2}+\|\nabla \cdot \mathbf{z}\|^{2}+\left\|c_{\tau}\right\|^{2}+\|c\|_{1}^{2}\right) d t\right\} .
\end{aligned}
$$

Noting that the assumption $\Delta t^{n} \geq K_{2} h^{\frac{3}{2}}$ implies, for sufficiently small $h$,

$$
\frac{\alpha_{0}}{2} \Delta t^{n}-K h^{2} \geq\left(1-\frac{2 K}{\alpha_{0} K_{2}} h^{\frac{1}{2}}\right) \frac{\alpha_{0}}{2} \Delta t^{n} \geq \frac{\alpha_{0}}{3} \Delta t^{n},
$$

and adding, then applying Gronwall's lemma and $c_{h}(0)=R_{h} c(0)$ to yield

$$
\begin{aligned}
& \|\tilde{e}\|_{L^{\infty}\left(L^{2}\right)}+\mid\|\boldsymbol{\xi}\|_{L^{2}\left(L_{\varepsilon}^{2}\right)} \leq Q\left\{h^{\frac{3}{2}}\left(\|c\|_{L^{\infty}\left(H^{3}\right)}+\|c\|_{H^{1}\left(H^{2}\right)}\right)\right. \\
& \quad+\Delta t\left(\left\|(\nabla \cdot \mathbf{z})_{\tau}\right\|_{L^{2}\left(L^{2}\right)}+\|\nabla \cdot \mathbf{z}\|_{L^{2}\left(L^{2}\right)}+\left\|c_{\tau}\right\|_{L^{2}\left(L^{2}\right)}+\|c\|_{L^{2}\left(H^{1}\right)}\right) .
\end{aligned}
$$

Combining (84) with the estimates for $\tilde{\rho}$ in Lemma 3.3 and applying the triangle inequality we reach the conclusion of the theorem. 


\section{Numerical Experiments}

In this section we perform numerical experiments to verify the theoretically proven $\varepsilon$-uniform optimal-order convergence. The data for the test example is chosen as follows:

$$
\Omega=[0,1]^{d}, d=1,2, \Lambda=[0,1], \mathbf{v}=(1,1)^{T}, D=1, c_{0}=0 .
$$

The analytic solution is prescribed to be

$$
c(\mathbf{x}, t)=16 \sin t(\mathbf{1}-\mathbf{x}) \mathbf{x}\left(\frac{1}{2}+\frac{1}{\pi} \arctan \left(2 \varepsilon^{-\frac{1}{2}}\left(\frac{1}{16}-\left(\mathbf{x}-\frac{\mathbf{1}}{\mathbf{2}}\right)^{2}\right)\right)\right) .
$$

For convenient, we use $\mathbf{1}=(1,1)^{T}$ and $\frac{\mathbf{1}}{\mathbf{2}}=\left(\frac{1}{2}, \frac{1}{2}\right)^{T}$ here. Inserting the above expressions into equation (1a) yields the right term $f(\mathbf{x}, t)$.

In our numerical experiments, we choose $\varepsilon=0.001,0.0005,0.0001,0.00005$ respectively and use the lowest-order Raviart-Thomas rectangle mixed finite element to fit the convergence rate in our error estimates

$$
\begin{gathered}
\left\|c-c_{h}\right\|_{L^{\infty}\left(L^{2}\right)} \leq Q_{1} h+Q_{2} \Delta t, \\
\left\|c-\tilde{c}_{h}\right\|_{L^{\infty}\left(L^{2}\right)} \leq Q_{3} h^{\frac{3}{2}}+Q_{4} \Delta t \\
\left\|\mathbf{z}-\mathbf{z}_{h}\right\|_{L^{2}\left(L_{\varepsilon}^{2}\right)} \leq Q_{5} h+Q_{6} \Delta t .
\end{gathered}
$$

Here we perform computations with different $\varepsilon$ to test $\varepsilon$-uniform convergence for the characteristics-mixed finite element scheme and the postprocessing scheme for two-dimensional case. The results are presented in Tables 1-3. These results show that we nearly obtain the same constant for different $\varepsilon$, which suggests that these estimates do not depend explicitly on $\varepsilon$ as predicted by Theorem 4.1 and Theorem 4.2. These results also show that the the characteristics-mixed finite element scheme possesses the optimal order convergence rates both for the concentration and its flux, and the postprocessing scheme could enhance the spacial convergence order up to $\frac{3}{2}$ as predicted by Theorem 4.2 . 
Table 1: The convergence rate for $\left\|c-c_{h}\right\|$ in two-dimensional case

\begin{tabular}{ccccc}
\hline & $\varepsilon=0.001$ & $\varepsilon=0.0005$ & $\varepsilon=0.0001$ & $\varepsilon=0.00005$ \\
\hline$h=\Delta t$ & $\left\|c-c_{h}\right\|$ & $\left\|c-c_{h}\right\|$ & $\left\|c-c_{h}\right\|$ & $\left\|c-c_{h}\right\|$ \\
\hline $1 / 24$ & $5.1872 \mathrm{e}-004$ & $5.1552 \mathrm{e}-004$ & $5.1270 \mathrm{e}-004$ & $5.1929 \mathrm{e}-004$ \\
$1 / 32$ & $3.5419 \mathrm{e}-004$ & $3.9529 \mathrm{e}-004$ & $3.6124 \mathrm{e}-004$ & $3.8671 \mathrm{e}-004$ \\
$1 / 48$ & $2.5177 \mathrm{e}-004$ & $2.7292 \mathrm{e}-004$ & $2.7251 \mathrm{e}-004$ & $2.6193 \mathrm{e}-004$ \\
$1 / 64$ & $2.0144 \mathrm{e}-004$ & $2.1207 \mathrm{e}-004$ & $2.0169 \mathrm{e}-004$ & $2.0259 \mathrm{e}-004$ \\
\hline & & & convergence rate & 1.3262 \\
& & & constant & 0.1291 \\
\hline
\end{tabular}

Table 2: The convergence rate for $\left\|c-\tilde{c}_{h}\right\|$ in two-dimensional case

\begin{tabular}{ccccc}
\hline & $\varepsilon=0.001$ & $\varepsilon=0.0005$ & $\varepsilon=0.0001$ & $\varepsilon=0.00005$ \\
\hline$h=\Delta t$ & $\left\|c-c_{h}\right\|$ & $\left\|c-c_{h}\right\|$ & $\left\|c-c_{h}\right\|$ & $\left\|c-c_{h}\right\|$ \\
\hline $1 / 24$ & $3.0977 \mathrm{e}-006$ & $3.1249 \mathrm{e}-006$ & $3.0979 \mathrm{e}-006$ & $3.0976 \mathrm{e}-006$ \\
$1 / 32$ & $1.3440 \mathrm{e}-006$ & $1.3977 \mathrm{e}-006$ & $1.3221 \mathrm{e}-006$ & $1.3551 \mathrm{e}-006$ \\
$1 / 48$ & $6.9222 \mathrm{e}-007$ & $6.9221 \mathrm{e}-007$ & $6.9694 \mathrm{e}-007$ & $6.7333 \mathrm{e}-007$ \\
$1 / 64$ & $4.2514 \mathrm{e}-007$ & $4.3746 \mathrm{e}-007$ & $4.4894 \mathrm{e}-006$ & $4.3429 \mathrm{e}-007$ \\
\hline & & & convergence rate & 1.5952 \\
& & & constant & 0.0024 \\
\hline
\end{tabular}

Table 3: The convergence rate for $\left\|\mathbf{z}-\mathbf{z}_{h}\right\|_{L_{\varepsilon}^{2}}$ in two-dimensional case

\begin{tabular}{ccccc}
\hline & $\varepsilon=0.001$ & $\varepsilon=0.0005$ & $\varepsilon=0.0001$ & $\varepsilon=0.00005$ \\
\hline$h=\Delta t$ & $\left\|\mathbf{z}-\mathbf{z}_{h}\right\|$ & $\left\|\mathbf{z}-\mathbf{z}_{h}\right\|$ & $\left\|\mathbf{z}-\mathbf{z}_{h}\right\|$ & $\left\|\mathbf{z}-\mathbf{z}_{h}\right\|$ \\
\hline $1 / 24$ & 0.0110 & 0.0104 & 0.0101 & 0.0110 \\
$1 / 32$ & 0.0086 & 0.0083 & 0.0083 & 0.0089 \\
$1 / 48$ & 0.0059 & 0.0058 & 0.0059 & 0.0055 \\
$1 / 64$ & 0.0045 & 0.0045 & 0.0049 & 0.0045 \\
\hline \multicolumn{3}{c}{ convergence rate } & 1.0144 \\
& & & constant & 0.7474 \\
\hline
\end{tabular}




\section{References}

[1] Arbogast, T. \& Wheeler, F. M. (1995) A characteristicsmixed finite element method for advection-dominated transport problems. SIAM J. Numer. Anal., 32, 404-424.

[2] Bause, M. \& Knabner, P. (2002) Uniform error analysis for Lagrange-Galerkin approximations of convection-dominated problems. SIAM J. Numer. Anal., 39, 1954-1984.

[3] Chen, H. Z., Zhou, Z. J., Wang H. \& Man H. Y. (2011) An optimal-order error estimate for a family of characteristic-mixed methods to transient convection-diffusion problems. Discrete Contin. Dyn. Syst. Ser. B, 15, 325-341.

[4] Ciarlet, P. G. (1978) The finite element method for elliptic problems. Amsterdam: North-Holland.

[5] Devore, R. A. \& Lorentz G. G. (1993) Constructive approximation. Berlin: Springer.

[6] Douglas, J. \& Russell, T. F. (1982) Numerical methods for convection-dominated diffusion problems based on combining the method of characteristics with finite element or finite difference procedures. SIAM J. Numer. Anal., 19, 871-885.

[7] Evans, L. C. (1998) Partial differential equations. Grad. Stud. Math.. Providence, RI: American Mathematics Society, 19.

[8] Ewing, R. E., Russell, T. F. \& Wheeler, M. F. (1984) Convergence analysis of an approximation of miscible displacement in porous media by mixed finite elements and a modified method of characteristics. Comput. Methods Appl. Mech. Engrg., 47, 73-92.

[9] Helmig, R. (1997) Multiphase flow and transport processes in the subsurface. Berlin: Springer-Verlag.

[10] Suli,E.(1988) Convergence and nonlinear stability of the LagrangeGalerkin method for the Navier-Stokes equations. Numer. Math., 53, no. 4, 459-483. 
[11] Morton, K.W., Priestley, A., Suli, E.(1988) Stability of the Lagrange-Galerkin method with nonexact integration. RAIRO Modl. Math. Anal. Numr. 22, no. 4, 625-653.

[12] Pironneau, O.(1981/82) On the transport-diffusion algorithm and its applications to the Navier-Stokes equations. Numer. Math., 38 , no. 3, 309-332.

[13] Raviat, P. A. \& Thomas, J. M. (1977) A mixed finite element methods for 2nd order elliptic problems. Mathmatical Aspects of the Finite Element Method, Lecture Notes in Maths. Berlin: Springer, 606, 292-315.

[14] Rios, H. -G., Stones, M. \& Tobiska, L. (1996) Numerical Methods for Singularly Perturbed Differential Equations. Springer Ser. Comput. Math.. Berlin: Springer, 24.

[15] RuI, H. X. (2002) Symmetic mixed covolume methods for parablic problems. Numer. Methods Partial Differential Eq., 18, 561-583.

[16] WANG, H. \& WANG, K. (2007) Uniform estimates for EulerianLagrangian methods for singularly perturbed time-dependent problems. SIAM J. Number. Anal., 45, 1305-1329.

[17] Wang, K. \& WANG, H. (2008) A uniform estimate for the ELLAM scheme for transport equations. Numer. Methods for PDEs., 24, 535554 .

[18] WANG, K. \& WANG, K. (2010) A uniform estimate for the MMOC for two-dimensional advection-diffusion equations. Numer. Methods for PDEs., 26, 1054-1069.

[19] Wang, K. \& Wang, H. (2011) Uniform estimates for a family of Eulerian-Lagrangian methods for time-dependent convection-diffusion equations with degenerate diffusion. IMA J. Numer. Anal., 31, 10061037. 


\section{Appendix A. The proof of Lemma 3.2}

Proof We decompose the left-hand side of (20) in the following form

$$
\begin{aligned}
\left(D^{-1}\left(\mathbf{w}-\Pi_{h} \mathbf{w}\right), \boldsymbol{\chi}\right)_{\Omega_{i, j}} & \\
= & \left(\left(D^{-1}(x, y)-D^{-1}\left(x_{i-\frac{1}{2}}, y_{j-\frac{1}{2}}\right)\right)\left(\mathbf{w}-\Pi_{h} \mathbf{w}\right), \boldsymbol{\chi}\right)_{\Omega_{i, j}} \\
& +\left(D^{-1}\left(x_{i-\frac{1}{2}}, y_{j-\frac{1}{2}}\right)\left(\mathbf{w}-\Pi_{h} \mathbf{w}\right), \boldsymbol{\chi}-\boldsymbol{\chi}\left(x_{i-\frac{1}{2}}, y_{j-\frac{1}{2}}\right)\right)_{\Omega_{i, j}} \\
& +\left(D^{-1}\left(x_{i-\frac{1}{2}}, y_{j-\frac{1}{2}}\right)\left(\mathbf{w}-\Pi_{h} \mathbf{w}\right), \boldsymbol{\chi}\left(x_{i-\frac{1}{2}}, y_{j-\frac{1}{2}}\right)\right)_{\Omega_{i, j}}
\end{aligned}
$$

in which the three terms on the right-hand side will be bounded term by term as follows.

The first term, which is much easy to estimate, is bounded by directly using (19) and Hölder inequality,

$$
\begin{aligned}
& \left|\left(\left(D^{-1}(x, y)-D^{-1}\left(x_{i-\frac{1}{2}}, y_{j-\frac{1}{2}}\right)\right)\left(\mathbf{w}-\Pi_{h} \mathbf{w}\right), \boldsymbol{\chi}\right)_{\Omega_{i, j}}\right| \\
& \quad \leq Q h^{2}\|\mathbf{w}\|_{1, \Omega_{i, j}}\|\chi\|_{\Omega_{i, j}}
\end{aligned}
$$

with $Q=Q\left(\left\|D^{-1}\right\|_{1, \infty}\right)$.

Let $D^{-1}=\left(D_{i j}^{-1}\right)_{2 \times 2}$ and $\chi=\left(\chi_{1}, \chi_{2}\right)^{T}$, then the second term is regrouped in the following manner,

$$
\begin{aligned}
\left(D^{-1}\left(x_{i-\frac{1}{2}}, y_{j-\frac{1}{2}}\right)\left(\mathbf{w}-\Pi_{h} \mathbf{w}\right), \boldsymbol{\chi}-\boldsymbol{\chi}\left(x_{i-\frac{1}{2}}, y_{j-\frac{1}{2}}\right)\right)_{\Omega_{i, j}} \\
=\left(D_{11}^{-1}\left(x_{i-\frac{1}{2}}, y_{j-\frac{1}{2}}\right)\left(w_{1}-\Pi_{i x} w_{1}\right), \chi_{1}-\chi_{1}\left(x_{i-\frac{1}{2}}, y_{j-\frac{1}{2}}\right)\right)_{\Omega_{i, j}} \\
\quad+\left(D_{22}^{-1}\left(x_{i-\frac{1}{2}}, y_{j-\frac{1}{2}}\right)\left(w_{2}-\Pi_{j y} w_{2}\right), \chi_{2}-\chi_{2}\left(x_{i-\frac{1}{2}}, y_{j-\frac{1}{2}}\right)\right)_{\Omega_{i, j}} \\
\quad+\left(D_{21}^{-1}\left(x_{i-\frac{1}{2}}, y_{j-\frac{1}{2}}\right)\left(w_{1}-\Pi_{i x} w_{1}\right), \chi_{2}-\chi_{2}\left(x_{i-\frac{1}{2}}, y_{j-\frac{1}{2}}\right)\right)_{\Omega_{i, j}} \\
\quad+\left(D_{12}^{-1}\left(x_{i-\frac{1}{2}}, y_{j-\frac{1}{2}}\right)\left(w_{2}-\Pi_{j y} w_{2}\right), \chi_{1}-\chi_{1}\left(x_{i-\frac{1}{2}}, y_{j-\frac{1}{2}}\right)\right)_{\Omega_{i, j}} .
\end{aligned}
$$

For the $D_{11}^{-1}$-term on the right-hand side of (.3), we use Taylor's expansion

$$
\chi_{1}(x, y)-\chi_{1}\left(x_{i-\frac{1}{2}}, y_{j-\frac{1}{2}}\right)=\chi_{1 x}(x, y)\left(x-x_{i-\frac{1}{2}}\right)
$$

to obtain

$$
\begin{aligned}
& \left(D_{11}^{-1}\left(x_{i-\frac{1}{2}}, y_{j-\frac{1}{2}}\right)\left(w_{1}-\Pi_{i x} w_{1}\right), \chi_{1}-\chi_{1}\left(x_{i-\frac{1}{2}}, y_{j-\frac{1}{2}}\right)\right)_{\Omega_{i, j}} \\
& \quad=D_{11}^{-1}\left(x_{i-\frac{1}{2}}, y_{j-\frac{1}{2}}\right)\left(\int_{\Omega_{i, j}} w_{1}(x, y) \chi_{1 x}(x, y)\left(x-x_{i-\frac{1}{2}}\right) d x d y\right. \\
& \left.\quad-\int_{\Omega_{i, j}} \Pi_{i x} w_{1}(x, y) \chi_{1 x}(x, y)\left(x-x_{i-\frac{1}{2}}\right) d x d y\right)
\end{aligned}
$$


Applying the definition of $\bar{w}_{1}, \Pi_{i x} w_{1}$ and the inverse inequality to bound the integral,

$$
\begin{aligned}
& \int_{\Omega_{i, j}} w_{1}(x, y) \chi_{1 x}\left(x-x_{i-\frac{1}{2}}\right) d x d y-\int_{\Omega_{i, j}} \Pi_{i x} w_{1}(x, y) \chi_{1 x}\left(x-x_{i-\frac{1}{2}}\right) d x d y \\
& =\int_{\Omega_{i, j}} w_{1}(x, y) \chi_{1 x}\left(x-x_{i-\frac{1}{2}}\right) d x d y \\
& -\frac{1}{\Delta x} \int_{\Omega_{i, j}}\left(x_{i}-x\right) \bar{w}_{1}\left(x_{i-1}, y\right) \chi_{1 x}\left(x-x_{i-\frac{1}{2}}\right) d x d y \\
& -\frac{1}{\Delta x} \int_{y_{j-1}}^{y_{j}} \bar{w}_{1}\left(x_{i}, y\right) \chi_{1 x} \int_{x_{i-1}}^{x_{i}}\left(x-x_{i-1}\right)\left(x-x_{i-\frac{1}{2}}\right) d x d y \\
& =\int_{\Omega_{i, j}} w_{1}(x, y) \chi_{1 x}\left(x-x_{i-\frac{1}{2}}\right) d x d y+\frac{(\Delta x)^{2} \Delta y}{12}\left(\bar{w}_{1}\left(x_{i-1}, y\right) \chi_{1 x}-\bar{w}_{1}\left(x_{i}, y\right)\right) \chi_{1 x} \\
& =\int_{\Omega_{i, j}} w_{1}(x, y) \chi_{1 x}\left(x-x_{i-\frac{1}{2}}\right) d x d y-\frac{(\Delta x)^{2}}{12} \int_{y_{j-1}}^{y_{j}}\left(w_{1}\left(x_{i}, y\right)-w_{1}\left(x_{i-1}, y\right)\right) d y \chi_{1 x} \\
& =\int_{\Omega_{i, j}} \int_{x_{i-\frac{1}{2}}}^{x}\left(w_{1 \alpha}(\alpha, y)-w_{1 \alpha}\left(x_{i-\frac{1}{2}}, y\right)\right) d \alpha \chi_{1 x}\left(x-x_{i-\frac{1}{2}}\right) d x d y \\
& -\int_{\Omega_{i, j}} \int_{x_{i-\frac{1}{2}}}^{x} w_{1 \alpha}\left(x_{i-\frac{1}{2}}, y\right) d \alpha\left(x-x_{i-\frac{1}{2}}\right) \chi_{1 x} d x d y-\frac{(\Delta x)^{2}}{12} \int_{\Omega_{i, j}} w_{1 x}(x, y) \chi_{1 x} d x d y \\
& =\int_{\Omega_{i, j}} \int_{x_{i-\frac{1}{2}}}^{x} \int_{x_{i-\frac{1}{2}}}^{\alpha} w_{1 \gamma \gamma}(\gamma, y) d \gamma d \alpha \chi_{1 x}\left(x-x_{i-\frac{1}{2}}\right) d x d y \\
& -\int_{\Omega_{i, j}} w_{1 x}\left(x_{i-\frac{1}{2}}, y\right)\left(x-x_{i-\frac{1}{2}}\right)^{2} \chi_{1 x} d x d y-\frac{(\Delta x)^{2}}{12} \int_{\Omega_{i, j}} w_{1 x}(x, y) \chi_{1 x} d x d y \\
& =\int_{\Omega_{i, j}} \int_{x_{i-\frac{1}{2}}}^{x} \int_{x_{i-\frac{1}{2}}}^{\alpha} w_{1 \gamma \gamma}(\gamma, y) d \gamma d \alpha \chi_{1 x}\left(x-x_{i-\frac{1}{2}}\right) d x d y \\
& +\frac{(\Delta x)^{3}}{12} \int_{y_{j-1}}^{y_{j}} \chi_{1 x} w_{1 x}\left(x_{i-\frac{1}{2}}, y\right) d y-\frac{(\Delta x)^{2}}{12} \int_{\Omega_{i, j}} w_{1 x}(x, y) \chi_{1 x} d x d y \\
& =\int_{\Omega_{i, j}} \int_{x_{i-\frac{1}{2}}}^{x} \int_{x_{i-\frac{1}{2}}}^{\alpha} w_{1 \gamma \gamma}(\gamma, y) d \gamma d \alpha \chi_{1 x}\left(x-x_{i-\frac{1}{2}}\right) d x d y \\
& -\frac{(\Delta x)^{2}}{12} \int_{\Omega_{i, j}}^{2}\left(w_{1 x}(x, y)-w_{1 x}\left(x_{i-\frac{1}{2}}, y\right) \chi_{1 x} d x d y\right. \\
& =\int_{\Omega_{i, j}} \int_{x_{i-\frac{1}{2}}}^{x} \int_{x_{i-\frac{1}{2}}}^{\alpha} w_{1 \gamma \gamma}(\gamma, y) d \gamma d \alpha \chi_{1 x}\left(x-x_{i-\frac{1}{2}}\right) d x d y \\
& -\frac{(\Delta x)^{2}}{12} \int_{\Omega_{i, j}}^{2} \int_{x_{i-\frac{1}{2}}}^{x^{2}} w_{1 \alpha \alpha}(\alpha, y) d \alpha \chi_{1 x} d x d y \\
& \leq Q h^{2}\left\|w_{1}\right\|_{2, \Omega_{i, j}}\left\|\chi_{1}\right\|_{\Omega_{i, j}} .
\end{aligned}
$$

Therefore the $D_{11}^{-1}$-term on the right-hand side of (.3) is bounded by

$$
\left(D_{11}^{-1}\left(x_{i-\frac{1}{2}}, y_{j-\frac{1}{2}}\right)\left(w_{1}-\Pi_{i x} w_{1}\right), \chi_{1}-\chi_{1}\left(x_{i-\frac{1}{2}}, y_{j-\frac{1}{2}}\right)\right)_{\Omega_{i, j}} \leq Q h^{2}\left\|w_{1}\right\|_{2, \Omega_{i, j}}\left\|\chi_{1}\right\|_{\Omega_{i, j}} .
$$

Similarly, the $D_{22}^{-1}$-term on the right-hand side of (.3) is bounded by

$$
\left(D_{22}^{-1}\left(x_{i-\frac{1}{2}}, y_{j-\frac{1}{2}}\right)\left(w_{2}-\Pi_{j y} w_{2}, \chi_{2}-\chi_{2}\left(x_{i-\frac{1}{2}}, y_{j-\frac{1}{2}}\right)\right)\right)_{\Omega_{i, j}} \leq Q h^{2}\left\|\chi_{2}\right\|_{\Omega_{i, j}}\left\|w_{2}\right\|_{2, \Omega_{i, j}} \text {. }
$$

We notice that the $D_{12}^{-1}$ and $D_{21}^{-1}$ terms on the right-hand side of (.3) satisfy a kind of symmetry, so we only estimate the $D_{21}^{-1}$-term here. By taking the following Taylor's expansion

$$
\chi_{2}(x, y)-\chi_{2}\left(x_{i-\frac{1}{2}}, y_{j-\frac{1}{2}}\right)=\chi_{2 y}\left(y-y_{j-\frac{1}{2}}\right),
$$




$$
w_{1}(x, y)=w_{1}\left(x, y_{j-\frac{1}{2}}\right)+w_{1 y}\left(x, y_{j-\frac{1}{2}}\right)\left(y-y_{j-\frac{1}{2}}\right)+\int_{y_{j-\frac{1}{2}}}^{y}(y-\beta) w_{1 \beta \beta}(x, \beta) d \beta
$$

into the $D_{21}^{-1}$-term we obtain

$$
\begin{aligned}
& \left(D_{21}^{-1}\left(x_{i-\frac{1}{2}}, y_{j-\frac{1}{2}}\right)\left(w_{1}-\Pi_{i x} w_{1}\right), \chi_{2}-\chi_{2}\left(x_{i-\frac{1}{2}}, y_{j-\frac{1}{2}}\right)\right)_{\Omega_{i, j}} \\
& =\int_{\Omega_{i, j}} D_{21}^{-1}\left(x_{i-\frac{1}{2}}, y_{j-\frac{1}{2}}\right)\left(w_{1}\left(x, y_{j-\frac{1}{2}}\right)-\Pi_{i x} w_{1}\right) \chi_{2 y}\left(y-y_{j-\frac{1}{2}}\right) d x d y \\
& \quad+\frac{(\Delta y)^{3}}{12} \int_{x_{i-1}}^{x_{i}} D_{21}^{-1}\left(x_{i-\frac{1}{2}}, y_{j-\frac{1}{2}}\right) w_{1 y}\left(x, y_{j-\frac{1}{2}}\right) \chi_{2 y} d x d y \\
& \quad+\int_{\Omega_{i, j}} D_{21}^{-1}\left(x_{i-\frac{1}{2}}, y_{j-\frac{1}{2}}\right) \int_{y_{j-\frac{1}{2}}}^{y}(y-\beta) w_{1 \beta \beta}(x, \beta) d \beta \chi_{2 y}\left(y-y_{j-\frac{1}{2}}\right) d x d y
\end{aligned}
$$

in which the first integral vanishes since the mean value of $y-y_{j-\frac{1}{2}}$ is zero with the other integrands being constants, and the third integral can be bounded by $Q h^{2}\left\|w_{1 x x}\right\|_{\Omega_{i, j}}\left\|\chi_{2}\right\|_{\Omega_{i, j}}$ as done for (.4). To derive a bound for the second integral we need to consider it over the whole domain $\Omega$ so that we can apply the periodic boundary condition. Hence we bound the second integral in the following way,

$$
\begin{aligned}
& \frac{(\Delta y)^{3}}{12}\left|\sum_{i, j} \int_{x_{i-1}}^{x_{i}} D_{21}^{-1}\left(x_{i-\frac{1}{2}}, y_{j-\frac{1}{2}}\right) w_{1 y}\left(x, y_{j-\frac{1}{2}}\right) \chi_{2 y} d x\right| \\
&= \frac{(\Delta y)^{2}}{12} \mid \sum_{i, j} \int_{x_{i-1}}^{x_{i}} D_{21}^{-1}\left(x_{i-\frac{1}{2}}, y_{j-\frac{1}{2}}\right) w_{1 y}\left(x, y_{j-\frac{1}{2}}\right)\left(\chi_{2}\left(x, y_{j}\right)-\chi_{2}\left(x, y_{j-1}\right) d x \mid\right. \\
&= \frac{(\Delta y)^{2}}{12} \mid \sum_{i=1}^{I} \int_{x_{i-1}}^{x_{i}}\left\{D_{21}^{-1}\left(x_{i-\frac{1}{2}}, y_{J-\frac{1}{2}}\right) w_{1 y}\left(x, y_{J-\frac{1}{2}}\right) \chi_{2}\left(x, y_{J}\right)\right. \\
&\left.-D_{21}^{-1}\left(x_{i-\frac{1}{2}}, y_{\frac{1}{2}}\right) w_{1 y}\left(x, y_{\frac{1}{2}}\right) \chi_{2}\left(x, y_{0}\right)\right\} d x \\
&-\sum_{i=1}^{I} \sum_{j=1}^{J-1} \int_{x_{i-1}}^{x_{i}}\left\{D_{21}^{-1}\left(x_{i-\frac{1}{2}}, y_{j+\frac{1}{2}}\right) w_{1 y}\left(x, y_{j+\frac{1}{2}}\right)\right. \\
&\left.-D_{21}^{-1}\left(x_{i-\frac{1}{2}}, y_{j-\frac{1}{2}}\right) w_{1 y}\left(x, y_{j-\frac{1}{2}}\right)\right\} \chi_{2}\left(x, y_{j}\right) d x \mid
\end{aligned}
$$




$$
\begin{aligned}
\leq & \frac{(\Delta y)^{2}}{12} \mid \sum_{i=1}^{I}\left\{D_{21}^{-1}\left(x_{i-\frac{1}{2}}, y_{J-\frac{1}{2}}\right) w_{1 y}\left(x, y_{J-\frac{1}{2}}\right) \chi_{2}\left(x, y_{J}\right)\right. \\
& \left.-D_{21}^{-1}\left(x_{i-\frac{1}{2}}, y_{\frac{1}{2}}\right) w_{1 y}\left(x, y_{\frac{1}{2}}\right) \chi_{2}\left(x, y_{0}\right)\right\} d x \mid \\
& +\frac{(\Delta y)^{2}}{12} \mid \sum_{i=1}^{I} \sum_{j=1}^{J-1} \int_{x_{i-1}}^{x_{i}}\left\{D_{21}^{-1}\left(x_{i-\frac{1}{2}}, y_{j+\frac{1}{2}}\right) w_{1 y}\left(x, y_{j+\frac{1}{2}}\right)\right. \\
= & \left.\frac{(\Delta y)^{2}}{12} \mid \sum_{i=1}^{I} \int_{x_{i-1}}^{x_{i}} \int_{y_{\frac{1}{2}}}^{y_{J-\frac{1}{2}}}\left(D_{21}^{-1}\left(x_{i-\frac{1}{2}}, y_{j-\frac{1}{2}}\right) w_{1 y}\left(x, y_{j-\frac{1}{2}}\right)\right\} \chi_{2}(x, y)\right)_{y} \chi_{2}(x, c) d y d x \mid \\
& +\frac{(\Delta y)^{2}}{12}\left|\sum_{i=1}^{I} \sum_{j=1}^{J-1} \int_{x_{i-1}}^{x_{i}} \int_{y_{i-\frac{1}{2}}}^{y_{i+\frac{1}{2}}}\left(D_{21}^{-1}\left(x_{i-\frac{1}{2}}, y\right) w_{1 y}(x, y)\right)_{y} \chi_{2}\left(x, y_{j}\right) d y d x\right| \\
\leq & Q h^{2}\left\|\chi_{2}\right\|\left\|w_{1}\right\|_{2} .
\end{aligned}
$$

Where the periodic boundary condition and the equivalence between the discrete and continuous $L^{2}$ norm for $\chi_{2}$ are used. So we have bounded the $D_{21}^{-1}$ and $D_{12}^{-1}$ terms of $(.3)$ by $Q h^{2}\left\|\chi_{2}\right\|\left\|w_{1}\right\|_{2}$.

Collecting the estimates for the four terms on the right-hand side of (.3) to finish the bounding for the second term on the right-hand side of (.1)

$$
\sum_{i, j}\left(D^{-1}\left(x_{i-\frac{1}{2}}, y_{j-\frac{1}{2}}\right)\left(\mathbf{w}-\Pi_{h} \mathbf{w}\right), \boldsymbol{\chi}-\boldsymbol{\chi}\left(x_{i-\frac{1}{2}}, y_{j-\frac{1}{2}}\right)\right)_{\Omega_{i, j}} \leq Q h^{2}\|\chi\|\|\mathbf{w}\|_{2}
$$

What remains is to estimate the third term on the right-hand side of (.1). We decompose this term as follows

$$
\begin{aligned}
\left(D^{-1}\right. & \left.\left(x_{i-\frac{1}{2}}, y_{j-\frac{1}{2}}\right)\left(\mathbf{w}-\Pi_{h} \mathbf{w}\right), \chi\left(x_{i-\frac{1}{2}}, y_{j-\frac{1}{2}}\right)\right)_{\Omega_{i, j}} \\
= & \left(D_{11}^{-1}\left(x_{i-\frac{1}{2}}, y_{j-\frac{1}{2}}\right)\left(w_{1}-\Pi_{i x} w_{1}\right), \chi_{1}\left(x_{i-\frac{1}{2}}, y_{j-\frac{1}{2}}\right)\right)_{\Omega_{i, j}} \\
& +\left(D_{22}^{-1}\left(x_{i-\frac{1}{2}}, y_{j-\frac{1}{2}}\right)\left(w_{2}-\Pi_{j y} w_{2}\right), \chi_{2}\left(x_{i-\frac{1}{2}}, y_{j-\frac{1}{2}}\right)\right)_{\Omega_{i, j}} \\
& +\left(D_{21}^{-1}\left(x_{i-\frac{1}{2}}, y_{j-\frac{1}{2}}\right)\left(w_{1}-\Pi_{i x} w_{1}\right), \chi_{2}\left(x_{i-\frac{1}{2}}, y_{j-\frac{1}{2}}\right)\right)_{\Omega_{i, j}} \\
& +\left(D_{12}^{-1}\left(x_{i-\frac{1}{2}}, y_{j-\frac{1}{2}}\right)\left(w_{2}-\Pi_{j y} w_{2}\right), \chi_{1}\left(x_{i-\frac{1}{2}}, y_{j-\frac{1}{2}}\right)\right)_{\Omega_{i, j}} .
\end{aligned}
$$

We need only to estimate the first term on the right side of (.6) since the other three terms can be bounded similarly. Noting the definition (17) of $\bar{w}$ to have

$$
\int_{\Omega_{i, j}}\left(w_{1}-\Pi_{i x} w_{1}\right) d x d y=\int_{\Omega_{i, j}}\left(\bar{w}_{1}(x, y)-\Pi_{i x} w_{1}(x, y)\right) d x d y
$$


and plugging the following Taylor's expansions

$$
\begin{aligned}
& \bar{w}_{1}(x, y)=\bar{w}_{1}\left(x_{i-\frac{1}{2}}, y\right)+\bar{w}_{1 x}\left(x_{i-\frac{1}{2}}, y\right)\left(x-x_{i-\frac{1}{2}}\right)+\frac{1}{\Delta y} \int_{x_{i-\frac{1}{2}}}^{x} \int_{y_{j-1}}^{y_{j}}(x-\alpha) w_{1 \alpha \alpha}(\alpha, y) d y d \alpha \\
& \bar{w}_{1}\left(x_{i-1}, y\right)=\bar{w}_{1}\left(x_{i-\frac{1}{2}}, y\right)-\frac{\Delta x}{2} \bar{w}_{1 x}\left(x_{i-\frac{1}{2}}, y\right) d+\frac{1}{\Delta y} \int_{x_{i-1} x_{i-\frac{1}{2}}}^{y_{j}}\left(x-x_{i-1}\right) w_{1 x x}(x, y) d y d x \\
& \bar{w}_{1}\left(x_{i}, y\right)=\bar{w}_{1}\left(x_{i-\frac{1}{2}}, y\right)+\frac{\Delta x}{2} \bar{w}_{1 x}\left(x_{i-\frac{1}{2}}, y\right)+\frac{1}{\Delta y} \int_{x_{i-\frac{1}{2}}}^{x_{i}} \int_{y_{j-1}}^{y_{j}}\left(x_{i}-x\right) w_{1 x x}(x, y) d y d x
\end{aligned}
$$

into the expression of $\Pi_{i x} w_{1}(x, y)$ to rewrite it as follows,

$$
\begin{gathered}
\prod_{i x} w_{1}(x, y)=\bar{w}_{1}\left(x_{i-\frac{1}{2}}, y\right)+\bar{w}_{1 x}\left(x_{i-\frac{1}{2}}, y\right)\left(x-x_{i-\frac{1}{2}}\right) \\
\quad+\frac{x_{i}-x}{\Delta x \Delta y} \int_{x_{i-1}}^{x_{i-\frac{1}{2}}} \int_{y_{j-1}}^{y_{j}}\left(x-x_{i-1}\right) w_{1 x x}(x, y) d y d x \\
\quad+\frac{x-x_{i-1}}{\Delta x \Delta y} \int_{x_{i-\frac{1}{2}}}^{x_{i}} \int_{y_{j-1}}^{y_{j}}\left(x_{i}-x\right) w_{1 x x}(x, y) d y d x
\end{gathered}
$$

Then subtracting $\pi_{i x} \bar{w}_{1}$ form $\bar{w}_{1}$ and reusing the equivalence between the discrete and continuous $L^{2}$ norms to obtain

$$
\begin{aligned}
& \left(D_{11}^{-1}\left(x_{i-\frac{1}{2}}, y_{j-\frac{1}{2}}\right)\left(w_{1}-\Pi_{i x} w_{1}\right), \chi_{1}\left(x_{i-\frac{1}{2}}, y_{j-\frac{1}{2}}\right)\right)_{\Omega_{i, j}} \\
& =D_{11}^{-1}\left(x_{i-\frac{1}{2}}, y_{j-\frac{1}{2}}\right) \chi_{1}\left(x_{i-\frac{1}{2}}, y_{j-\frac{1}{2}}\right) \int_{\Omega_{i, j}} \frac{1}{\Delta y} \int_{x_{i-\frac{1}{2}}}^{y_{j}} \int_{y_{j-1}}^{y_{j-\frac{1}{2}}}(x-\alpha) w_{1 \alpha \alpha}(\alpha, y) d y d \alpha d x d y \\
& -D_{11}^{-1}\left(x_{i-\frac{1}{2}}, y_{j-\frac{1}{2}}\right) \chi_{1}\left(x_{i-\frac{1}{2}}, y_{j-\frac{1}{2}}\right) \int_{\Omega_{i, j}}^{y_{i}}\left(x-x_{i-1}\right) w_{1 x x}(x, y) d y d x d x d y \\
& -D_{11}^{-1}\left(x_{i-\frac{1}{2}}, y_{j-\frac{1}{2}}\right) \chi_{1}\left(x_{i-\frac{1}{2}}, y_{j-\frac{1}{2}}\right) \int_{\Omega_{i, j}}^{\frac{x_{i}}{\Delta x-x_{i-1}}} \frac{x}{\Delta x \Delta y} \int_{x_{i-\frac{1}{2}}}^{x_{j}} \int_{y_{j-1}}^{y_{j}}\left(x_{i}-x\right) w_{1 x x}(x, y) d y d x d x d y \\
& \leq Q h^{2}\|\chi\|_{\Omega_{i, j}}\left\|w_{1}\right\|_{2, \Omega_{i, j}},
\end{aligned}
$$

which implies that the third term on the right-hand side of (.1) can be bounded by

$$
\left(D^{-1}\left(x_{i-\frac{1}{2}}, y_{j-\frac{1}{2}}\right)\left(\mathbf{w}-\Pi_{h} \mathbf{w}\right), \boldsymbol{\chi}\left(x_{i-\frac{1}{2}}, y_{j-\frac{1}{2}}\right)\right)_{\Omega_{i, j}} \leq Q h^{2}\|\chi\|_{\Omega_{i, j}}\left\|w_{1}\right\|_{2, \Omega_{i, j}} .
$$

Combining (.2), (.5) with (.9), we prove (20).

This completes the proof of Lemma 3.2. 